\title{
PROGRAMAÇÃO MATEMÁTICA PARA AVALIAÇÃO DE VIABILIDADE ECONÔMICA DA PRODUÇÃO DE LEITE BOVINO EM PEQUENA ESCALA NO SUL DE GOIÁS
}

\author{
Rui Fonseca Veloso ${ }^{1}$ \\ Eliane Gonçalves Gomes ${ }^{2}$ \\ Luis Gustavo Barioni ${ }^{1}$ \\ Fernando Borges Fernandes ${ }^{3}$ \\ Homero Chaib Filho ${ }^{1}$ \\ Antônio Jorge de Oliveira ${ }^{2}$ \\ Ana Paula da Silva ${ }^{4}$ \\ Adriana Coloca ${ }^{5}$
}

Resumo: Este artigo apresenta um modelo bioeconômico para avaliação ex-ante de um empreendimento de pequena escala, que tem como atividade principal a produção de leite bovino na região Sul de Goiás. É proposto um modelo de programação linear multiperiódico, com horizonte de planejamento de 10 anos, que descreve o sistema de produção. O objetivo é avaliar a política de crédito rural com recursos públicos. Deseja-se saber se o empreendedor investiria e manter-se-ia na atividade, com uma taxa de juros $6 \%$ ao ano. Os resultados indicam que é possível assumir os compromissos decorrentes do financiamento, apesar da baixa renda líquida ao final do décimo ano.

Palavras-chave: Programação matemática; Viabilidade econômica; Modelo de fazenda.

Abstract: This paper presents a bioeconomic small-scale farm model to carryout ex ante investment analysis of dairy activity in the South of Goiás, Brazil. We propose a multiperiodic linear programming model, with 10 years of planning horizon, which describes the production system. The main objective is to evaluate the rural credit police with public resources. We want to know if the entrepreneur would invest and keep his the rural activity, paying an interest rate of $6 \%$ per year. The results indicate that it's possible to pay the financing, despite of the low net income in the 10th year.

Key words: Mathematical programming; Economic sustainability; Small scale farm model.

\footnotetext{
${ }^{1}$ Embrapa Cerrados \{rui; barioni; homero\}@cpac.embrapa.br

2 Embrapa Sede - SGE \{eliane.gomes; antonio.jorge\}@embrapa.br

${ }^{3}$ Embrapa Cerrados, autônomo

${ }^{4}$ Embrapa Cerrados, Fazenda Matinha, Silvânia-GO

${ }^{5}$ Fazenda Madeira, Gameleira-GO
} 


\section{INTRODUÇÃO}

Em países em desenvolvimento a produção agropecuária de pequena escala enfrenta mais restrições socioeconômicas do que aquelas de países desenvolvidos, protegidos com significativos aportes de créditos subsidiados (RUBEN, PENDER, 2004; KUYVENHOVEN, 2004; BERGER et al., 2006). Nesse contexto é importante a avaliação da viabilidade de medidas governamentais visando a sustentabilidade econômica da pequena agricultura no Brasil.

Estudar restrições para o crescimento de produção em propriedades rurais de pequena escala no Cerrado brasileiro é uma tarefa que se relaciona à escassez de diferentes recursos, à avaliação de tecnologias apropriadas, às dificuldades quanto às estruturas institucionais locais, à predominância de recursos humanos com baixa escolaridade e a outros aspectos, como o comportamento do produtor. A compreensão desses aspectos requer dados e informações relevantes sobre a dinâmica dos sistemas de produção, demandando análises com a participação do produtor rural.

Atendendo à solicitação de formuladores de políticas públicas, um sistema de produção de leite foi conceituado, a partir de uma realidade estudada, especificado e avaliado em termos de sustentabilidade bioeconômica. Partiu-se do pressuposto de que um empreendedor, possuidor de uma pequena área de terra, localizada na região sul goiana, deseja avaliar o investimento necessário para implantação da atividade de produção de leite bovino. O problema consiste em determinar se o empreendedor investiria e manter-se-ia na atividade, pagando uma taxa de $6 \%$ ao ano de juros do crédito rural com recursos públicos.

Este artigo apresenta um modelo bioeconômico para avaliação ex-ante de um empreendimento que tem como atividade principal a produção de leite bovino na região Sul de Goiás, com o objetivo de avaliar a política de crédito rural a uma taxa de juros de $6 \%$ a.a. para investimentos. Especificamente, o estudo visou projetar a atividade de produção de leite num horizonte de 20 semestres e avaliar a viabilidade econômica de investimentos na aquisição de vacas holandesas puras e/ou cruzadas e em infra-estrutura mínima para o empreendimento. Avaliou-se a captação de crédito para os custeios para a atividade pecuária e sub-atividades agrícolas, também à uma taxa de $6 \%$ a.a.

$\mathrm{Na}$ abordagem clássica, o desenvolvimento de modelos bioeconômicos faz-se a partir de levantamentos de dados de fazendas amostradas em uma determinada região, estabelecimento de fazendas típicas e contabilidade da margem bruta de cada atividade produtiva. Diferentemente, o modelo aqui apresentado foi construído a partir de uma compreensão, obtida através do estudo da dinâmica do negócio de uma fazenda envolvendo um sistema de produção de leite de pequena escala (BÖRNER, 2005). Considerou-se a economia regional em que a fazenda está incorporada e especificou-se o sistema estudado e a infra-estrutura mínima que estaria associada à escala de produção do empreendimento. Por último, foram apresentados resultados e comentários finais.

Com esse estudo espera-se oferecer uma ferramenta para síntese de conhecimentos, traduzidos na especificação de um modelo e dos dados e informações a ele incorporados, por meio de uma planilha eletrônica. Essa ferramenta computacional permitirá a troca de dados e informações entre pesquisadores, técnicos e produtores. A estrutura integrada de dados e informações aqui apresentadas visa melhorar a eficiência de transferência de dados, conhecimento e tecnologia entre os atores envolvidos.

\section{FONTE DE DADOS E METODOLOGIA}

Foi utilizado o método de estudo de caso recomendado por Maxwell (1986) para trabalhos de $\mathrm{P} \& \mathrm{D}$ de sistemas agropecuários envolvendo uma equipe 
multidisciplinar. Este modelo é bastante utilizado em pequenos e grandes negócios como meio de compreensão e análise de problemas. Tal método é ainda apoiado pela teoria de comportamento da fazenda-empresa discutida por Patrick, Eisgruber (1968), a qual refere-se a como mudanças nas características internas da fazenda, resultantes de mudanças na importância relativa de várias metas, levaria a gerência a responder diferentemente às mesmas condições em tempos diferentes. Assim, foram obtidos e usados dados e informações gerados a partir do monitoramento do negócio de uma fazenda na região Sul de Goiás e de críticas e sugestões de outros produtores e técnicos da área de produção animal.

Seguindo a visão clássica dos modelos de pesquisa operacional, a primeira fase em um processo de resolução de um problema de otimização é a sua estruturação, ou seja, a construção de um modelo matemático que represente, tanto objetivamente quanto quantitativamente, o problema a ser resolvido. Optou-se pela programação matemática como técnica apropriada para tratar esse problema da melhor alocação de escassos recursos para tal empreendimento. A razão deve-se ao fato de essa técnica possibilitar o desenvolvimento de um instrumento de fácil operacionalização, e com ele poder realizar avaliações de estratégias e cenários para melhorar a gestão no uso de tais recursos. Ela possibilita representar as regras de decisões de gerentes e proprietários rurais (HAZELL, NORTON, 1986).

No caso apresentado nesse artigo, o modelo de otimização incorpora como objetivo único a maximização de um fluxo de caixa multi-periódico, sujeito a um conjunto de restrições para estudar a política de investimentos na produção de leite bovino como uma atividade de pequena escala especializada, sem a complementaridade de outras como as produções de madeira e de grãos. Essas restrições estão relacionadas às limitações de recursos e alternativas técnicas. E, nesse caso específico, o modelo matemático desenvolvido é uma aproximação da dinâmica de uma fazenda como um negócio assumindo entre outros o pressuposto da linearidade.

Para o seu desenvolvimento, utilizouse de dados gerados a partir de um estudo de caso (uma fazenda) na região de interesse. Entretanto, a formulação de qualquer modelo matemático requer, na maioria dos estudos de pesquisa operacional, alguns parâmetros, que não foram previamente quantificados. $\mathrm{O}$ estudo de caso e a consulta a especialistas competentes possibilitou estimá-los e validá-los com a participação de técnicos e produtores rurais, que avaliaram soluções geradas pelo modelo, segundo estrutura da Figura 1, conforme apresentado em Vidal (2006). 


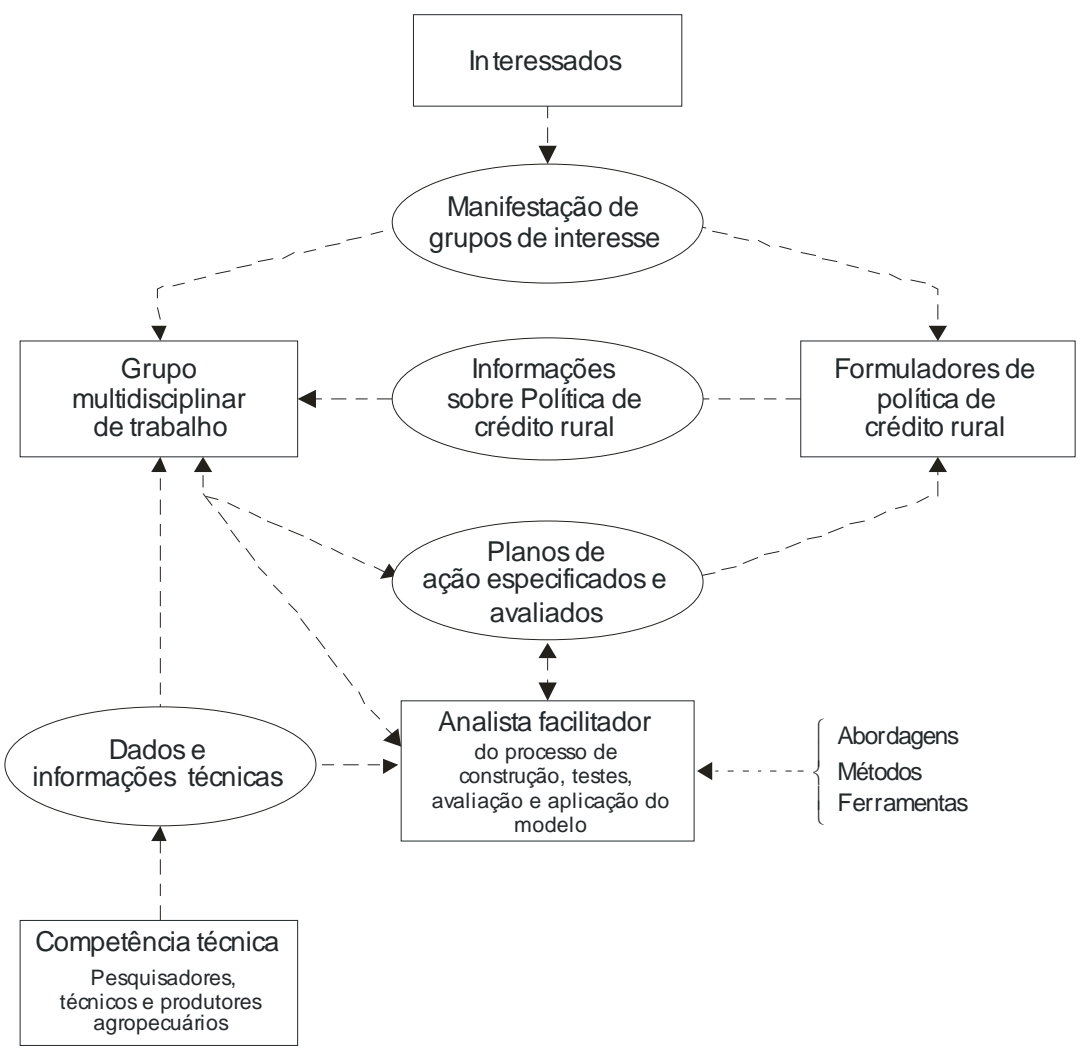

Figura 1: Baseada em abordagem de pesquisa operacional para resolução de problema (VIDAL, 2006). 
Um exemplo de tais parâmetros são os dados de produção de matéria seca, energia e fibra de cada alimento volumoso utilizado pelos animais e suas quantidades ofertadas nos períodos das chuvas e da seca. Muita pesquisa agrícola desenvolvida em estações experimentais é conduzida sob condições diferentes daquelas enfrentadas por produtores rurais. Em geral, adota-se uma abordagem reducionista que não gera todos os dados e informações importantes para um melhor entendimento da dinâmica dos processos incorporados em uma fazenda comercial. Isto tem sido enfatizado por Dent et. al. (1986) e Janssen, Goldsworthy (1996).

\subsection{Caracterização do sistema de produção}

A estrutura da unidade de produção de leite bovino a que se refere este estudo baseia-se no estudo do caso da Fazenda Matinha, localizada no Sul de Goiás, mais especificamente no município de Silvânia-GO (VELOSO et al., 2003). Trata-se de uma propriedade de pequena escala (61 hectares). Para desenvolver o modelo proposto, partiu-se da compreensão da dinâmica do referido sistema de produção, das especificações dos formuladores de política agrícola, de dados e de informações estabelecidas com a participação de produtores rurais, de técnicos especialistas e dos vários pressupostos estabelecidos no próximo sub-item.

A compreensão geral da dinâmica do sistema de produção de cada fazenda, em seus aspectos técnicos, foi conseguida através de entrevistas dirigidas ao produtor e à sua família, do processo de construção de um banco de dados, críticas e análises dos dados primários nele incorporados, bem como do acompanhamento das operações que compõem o processo produtivo como um todo. As visitas foram feitas com freqüência mensal até o final de 2004 e duas vezes durante o processo de construção do modelo. Para atender às necessidades de dados e informações da gerência de toda a fazenda foi criado um protótipo de um sistema de informações técnicas e gerenciais, o qual foi implantado com a participação do produtor e de sua família. Este sistema teve os propósitos de atender às necessidades de planejamento e gerência da fazenda, bem como de dar suporte aos demais esforços de $P \& D$ previstos na ampla estrutura de dados e informações de um projeto conduzido pela Embrapa Cerrados.

Em um primeiro momento foi realizada uma revisão no inventário patrimonial da propriedade, constando das instalações, benfeitorias, rebanho, maquinário e implementos em geral. A partir daí foi revisado o plano de contas e a contabilidade de custos, visando atender aos requerimentos de dados e informações para construção do modelo de fazenda.

O funcionamento do sistema de produção estudado requer um orçamento base de R\$ 43.882,56 e para o sistema de produção projetado $\mathrm{R} \$ 30.234,14$, conforme Tabela 1. Isto significa que o investidor terá que gerar uma receita que pague por todos os custos diretos mais aqueles associados à escala especificada de produção. A razão das diferenças entre valores dos centros de custos, mais os valores de depreciação ou reserva de capital para reposição de ativos, de cada sistema de produção apresentado na Tabela 1, baseia-se nas especificações dos formuladores ou analistas de política de crédito rural, clientes desse modelo de apoio à tomada de decisão. Vale mencionar que a Fazenda Matinha tem incorporadas em seu sistema de produção atividades como: quatro hectares de eucalyptus, alguns pequenos animais, dez hectares de soja e meio hectare de café. Assim, ela visa uma economia de escopo $^{6}$, que nesse caso é a complementaridade entre atividades de produção. E a questão de economia de escala ${ }^{7}$, em modelos de programação

\footnotetext{
6 "Economia de escopo ocorre sempre que o custo de produção conjunta é menor que o custo total de produção independente” (BAUMOL et al., 1988). 7 "Economia de escala é a propriedade pela qual o custo total médio no longo prazo cai á medida que a quantidade produzida aumenta” (MANKIW, 2001).
} 
linear para planejamento de fazenda, está bem demonstrada em Dent et al. (1986).

Discussões sobre economias de escala e de escopo, como apresentado em Oude Lansink, Stefanou (2001) demonstram que, embora muitos produtores holandeses de cultivos anuais tenham uma forte propensão à especialização, as melhores estratégias para alocação de suas áreas têm sido alcançadas graças ao sinergismo entre cultivos, o que permite redução de custos de produção. Da mesma forma, a complementaridade de atividades de produção de grãos e pecuária em um sistema (fazenda) integrado tem sido tecnicamente defendido por equipes de pesquisadores da Embrapa (VILELA et al., 2001). 
Tabela 1: Funcionamento dos sistemas de produção de leite bovino (estudado e projetado).

\begin{tabular}{|l|l|r|r|}
\cline { 2 - 4 } \multicolumn{1}{c|}{} & \multicolumn{1}{c|}{$\begin{array}{c}\text { Fazenda } \\
\text { Matinha }\end{array}$} & $\begin{array}{c}\text { Fazenda } \\
\text { projetada }\end{array}$ \\
\cline { 2 - 4 } \multicolumn{1}{c|}{} & $\begin{array}{l}\text { Área total do sistema de produção (incluindo } \\
\text { 20\% de reserva legal) }\end{array}$ & 61 ha & 63,13 ha \\
\hline 1MEq_y01 & Ano 01 Manutenção de equipamentos (R\$) & 648,00 & 648,00 \\
\hline 1MCa_y01 & Ano 01 Manutenção de automóvel (R\$) & $5.378,40$ & $2.689,20$ \\
\hline 1CFa_y01 & $\begin{array}{l}\text { Ano 01 Consumo básico da família ou salários } \\
\text { da mão-de-obra familiar (R\$) }\end{array}$ & $19.414,08$ & $13.000,00$ \\
\hline 1Cte_y01 & Ano 01 Consumo telefone (R\$) & $1.555,20$ & 622,08 \\
\hline 1CEn_y01 & Ano 01 Consumo energia (R\$) & $3.628,80$ & $3.628,80$ \\
\hline 1OC_y01 & Ano 01 Outros custos (R\$) & $6.778,08$ & $3.389,04$ \\
\hline 1RC_y01 & Ano 01 Reserva capital p/ repor ativos (R\$) & $6.480,00$ & $6.257,02$ \\
\hline & Sub-total (R\$) & $43.882,56$ & $30.234,14$ \\
\cline { 2 - 4 }
\end{tabular}


2.1.1. Pressupostos incorporados no modelo multiperiódico

Assumiu-se, entre outros pressupostos, que:

- a fazenda é uma propriedade privada;

- a fazenda dispõe de uma infraestrutura mínima, no âmbito da comunidade e do município, quanto aos recursos terra, mão-de-obra e capital de giro, e conta com uma política de crédito rural para empreendimentos de pequena escala (Tabela 2);

- o horizonte de planejamento de 10 anos com dois períodos anuais é adequado devido à necessidade de representar as mudanças nas quantidades de forragens produzidas nos períodos das águas e da seca, e do período de maior produção de uma vaca leiteira;

- a área total de terra disponível inclui 4,5 hectares para produção de milho para silagem e de milheto de maneira seqüencial, um hectare de cana, 5 hectares de pasto tanzânia rotacionado e 40 hectares de braquiária degradada;

- a forragem produzida por meio dos cultivos de cana e de braquiária são disponibilizadas para vacas mestiças;

- todo o esterco gerado com o manejo das vacas nas proximidades do curral é coletado e distribuído no cultivo de cana, e que nessa operação 5 diárias (de 8 horas cada uma) são anualmente contratadas a um custo de $\mathrm{R} \$$ 30,00/dia;

- é possível a compra de silagem de milho, aluguel de horas de trator e equipamentos de um produtor vizinho ou de um outro agente no mercado local (por exemplo, uma associação de produtores);

- a compra de silagem de um vizinho, associação ou cooperativa custa $\mathrm{R} \$$ 0,065 / kg;

- a associação ou cooperativa de produtores rurais tem mercado para a produção de crias (bezerros ou bezerras);

- a associação ou cooperativa de produtores participa do mercado de insumos, leite e outros produtos; 
Tabela 2: Dados parciais do sistema de produção.

\begin{tabular}{|c|c|c|}
\hline Áreas de terra & Unidade & Quantidade \\
\hline Ano 01 área abaixo estrada lavoura1 (área $a_{M i s)}$ & ha & 4,5 \\
\hline Ano 01 área abaixo estrada safrinha & ha & 4,5 \\
\hline Ano 01 Cana 01 (área ${ }_{C a}$ ) & ha & 1 \\
\hline Ano 01 Pasto 01 Tanzânia rotacionado (área $a_{T z}$ ) & ha & 5 \\
\hline Ano 01 Pasto 02 Braquiaria brizantha (área $a_{B r}$ ) & ha & 40 \\
\hline
\end{tabular}

\begin{tabular}{|l|c|c|}
\hline Infra-estrutura da fazenda & Unidade & Quantidade \\
\hline Curral e cercas & $\mathrm{R} \$$ & $20.000,00$ \\
\hline Ordenhadeira & $\mathrm{R} \$$ & $30.000,00$ \\
\hline Resfriador & $\mathrm{R} \$$ & $30.000,00$ \\
\hline Casas & $\mathrm{R} \$$ & $20.000,00$ \\
\hline Total & $\mathrm{R} \$$ & $\mathbf{1 0 0 . 0 0 0 , 0 0}$ \\
\hline
\end{tabular}

\begin{tabular}{|l|c|c|}
\hline Serviços de terceiros & Unidade & Quantidade \\
\hline Hora de trator com grade aradora ou pulverizador & $\mathrm{R} \$ /$ hora & 45,00 \\
\hline Hora de trator com ensiladeira & $\mathrm{R} \$ /$ hora & 55,00 \\
\hline Mão-de-obra contratada & $\mathrm{R} \$ /$ dia & 30,00 \\
\hline
\end{tabular}

\begin{tabular}{|c|c|c|c|}
\hline Crédito de custeio e capital de giro inicial & Unidade & Ano & Quantidade \\
\hline \multirow{10}{*}{$\begin{array}{l}\text { Ano } j \text { Empréstimo crédito de custeio pecuário(R\$) } \\
(V E C C P)\end{array}$} & R\$/ano & Ano 01 & $15.000,00$ \\
\hline & $\mathrm{R} \$$ /ano & Ano 02 & $15.000,00$ \\
\hline & R\$/ano & Ano 03 & $15.000,00$ \\
\hline & $\mathrm{R} \$ / \mathrm{ano}$ & Ano 04 & $15.000,00$ \\
\hline & $\mathrm{R} \$$ /ano & Ano 05 & $15.000,00$ \\
\hline & $\mathrm{R} \$$ ano & Ano 06 & $15.000,00$ \\
\hline & R\$/ano & Ano 07 & $15.000,00$ \\
\hline & $\mathrm{R} \$ / \mathrm{ano}$ & Ano 08 & $15.000,00$ \\
\hline & $\mathrm{R} \$ / \mathrm{ano}$ & Ano 09 & $15.000,00$ \\
\hline & $\mathrm{R} \$$ ano & Ano 10 & $15.000,00$ \\
\hline \multirow{10}{*}{$\begin{array}{l}\text { Ano j Empréstimo crédito de custeio lavoura (R\$) } \\
(V E C C L)\end{array}$} & $\mathrm{R} \$ / \mathrm{ano}$ & Ano 01 & $15.000,00$ \\
\hline & $\mathrm{R} \$ / \mathrm{ano}$ & Ano 02 & $15.000,00$ \\
\hline & $\mathrm{R} \$ / \mathrm{ano}$ & Ano 03 & $15.000,00$ \\
\hline & $\mathrm{R} \$ / \mathrm{ano}$ & Ano 04 & $15.000,00$ \\
\hline & R\$/ano & Ano 05 & $15.000,00$ \\
\hline & R\$/ano & Ano 06 & $15.000,00$ \\
\hline & R\$/ano & Ano 07 & $15.000,00$ \\
\hline & R\$/ano & Ano 08 & $15.000,00$ \\
\hline & R\$/ano & Ano 09 & $15.000,00$ \\
\hline & R\$/ano & Ano 10 & $15.000,00$ \\
\hline
\end{tabular}

\begin{tabular}{|l|c|c|}
\hline Capital de giro inicial & Unidade & Quantidade \\
\hline Ano 01 Capital caixa1_a em R\$ ano 1 (capital $)$ & $\mathrm{R} \$$ & $1.000,00$ \\
\hline
\end{tabular}


- as áreas de cana, de pastagem tanzânia e de pastagem braquiária encontram-se já implantadas;

- a área disponível para cultivos de milho e de milheto (em um processo rotacionado) encontra-se com bom potencial produtivo em decorrência de correções de fertilidade do solo e distribuição de chuvas favorável;

- além das 4.320 horas/ano disponibilizadas por 2 assalariados, é possível, para os trabalhos de rotina a contratação de mão-de-obra local (diarista) a um custo de R $\$ 30,00 /$ dia;

- tanto vacas holandesas (R\$ $1.500,00 /$ cabeça) de puro sangue ou mestiças (R\$ 1.200,00/cabeça) são compradas facilmente no mercado local;

- vacas de qualquer raça são descartadas ao valor de (R\$ $(600,00 * 0,95) /$ cabeça) ao final do sexto ano de produção;

- a taxa (5\%) de risco de morte de uma vaca em produção foi inserida no preço de venda da vaca descarte;

- as crias, independente da raça, são vendidas 6 meses após o nascimento gerando uma renda líquida média de R\$ 150,00/cria da raça holandesa e $\mathrm{R} \$ 100,00 /$ cria de raça cruzada ;

- não há problema de demanda decorrente da quantidade de leite que passará a ser produzido com o novo empreendimento;

- os 20 preços semestrais de um litro de leite, incorporados no modelo, variam em torno de aproximadamente $\mathrm{R} \$$ 0,40 , e são gerados aleatoriamente a partir da série histórica (ver gráficos apresentados em Anexo I e II e discussão em Veloso et al. (2003);

- o produtor tem crédito (de $\mathrm{R} \$$ $120.000,00$ a $6 \%$ a.a.) disponível para comprar até 80 vacas da raças holandesa ou cruzada;

- o crédito para custeio pecuário é de até $\mathrm{R} \$ 15.000,00$ a $6 \%$ a.a.;

- o crédito para custeio agrícola é de até $\mathrm{R} \$ 15.000,00$ a $10 \%$ ao ano;

- o produtor tem um crédito de R\$ $100.000,00$ a $6 \%$ a.a., disponível para investimentos em currais etc.; e 
o valor de sucata do investimento ( $\mathrm{R} \$$ 100.000,00) em infra-estrutura é estimado em $\mathrm{R} \$ 50.000,00$, no final do décimo ano.
- transferência de superávit ou déficit no caixa semestral (final do $2^{\circ}$ semestre de cada ano); e horizonte de planejamento de 10 anos.

\section{CONSTRUÇÃO DO MODELO MATEMÁTICO}

Neste estudo, o foco é sobre a atividade de produção leite, de tal maneira que as produções de forragem são sub-atividades. No modelo, considera-se a contratação de dias de trabalho de terceiros e uma reserva de capital concernente à depreciação de ativos do empreendimento. Em síntese, assume-se que o produtor deseja manter 0 critério de maximização de seus retornos econômicos, independente de preferências e crenças de membros de sua família (HARDAKER, 1979).

\subsection{Conceituação do modelo}

A conceituação do modelo baseia-se na estrutura apresentada na Figura 2 (adaptada de IBSNAT Project (1987)), com ênfase nos componentes:

- cultivos anuais e perenes (pastagens) para produção de forragens;

- rotação de culturas (milho e milheto) na mesma área durante o ano;

- investimentos em ativos como casas, currais e equipamentos;

- investimentos e desinvestimentos (descarte de vacas a preço de mercado) no rebanho;

- mão-de-obra familiar (2 pessoas com 3 meses de férias parceladas durante o ano);

- mão-de-obra contratada (diaristas);

- tratores alugados para preparação de solo para cultivos e preparação de silagem de milho;

- capital (R\$1.000,00) de giro inicial;

- crédito comercial eventual (Crédito do Produtor Rural a $20 \%$ a.a.);

- crédito rural a 6\% a.a.;

- conjunto de custos indiretos de produção (veja Tabela 1);

- produções e alocações de quantidades ofertadas de pastagens cultivadas nos períodos chuvoso $75 \%$ e seco $25 \%$;

- vendas semestrais leite;

ENGEVISTA, v. 9, n. 1, p. 57-81, junho 2007 


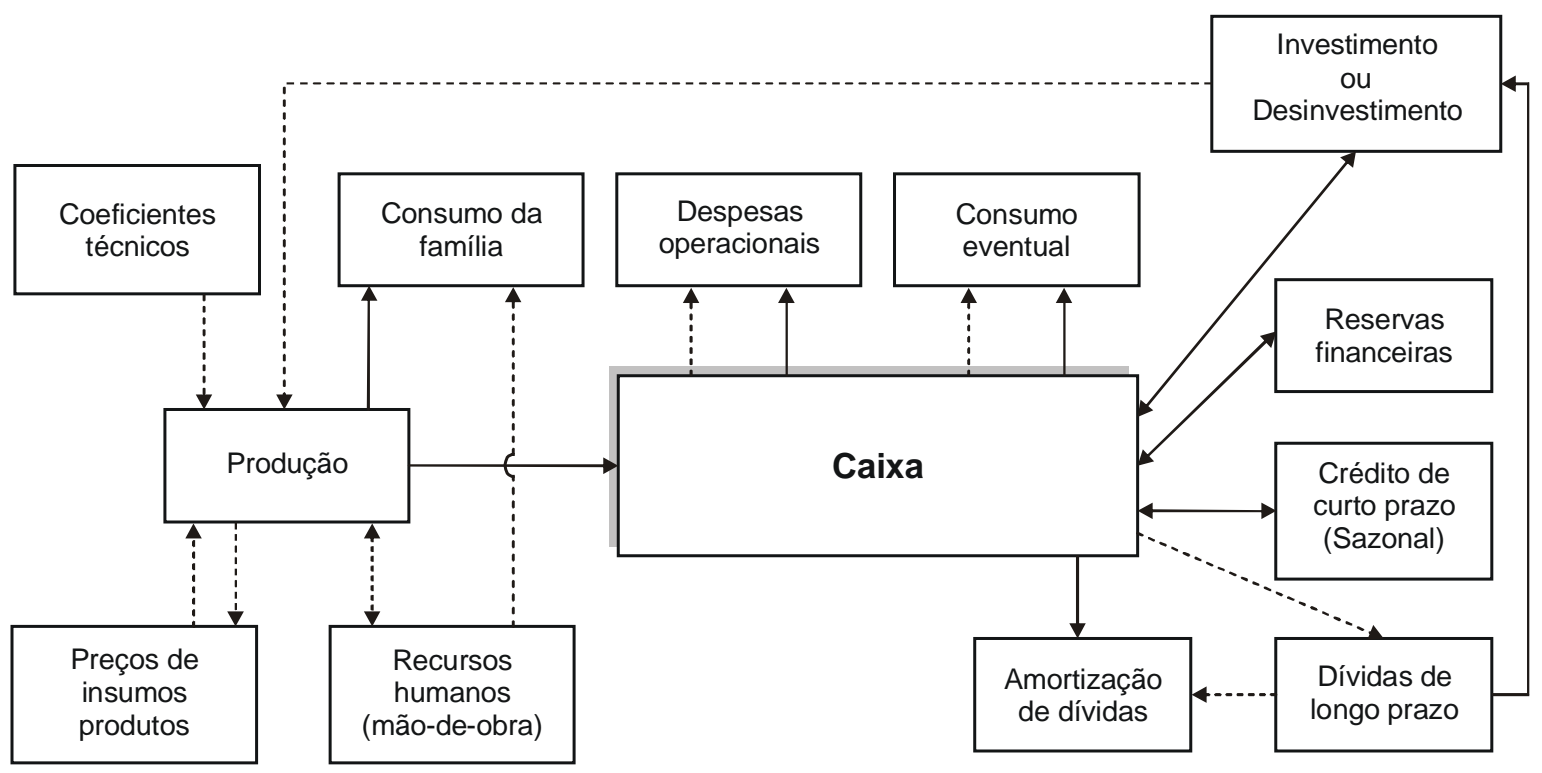

Figura 2: Baseado em modelo conceitual de fazenda como empresa, IBSNAT Project (1987). 


\subsection{Formulação do modelo}

Escolher o critério de decisão como sendo a maximização de retornos econômicos no médio prazo, adotado nesse estudo, foi uma racionalização do problema de sustentabilidade econômica de uma pequena unidade de produção de leite em Goiás.

A formulação completa do modelo matemático está sinteticamente descrita no Anexo III. Nela foram considerados $j$ períodos de tempo, $j=1 \ldots n$. A função objetivo visa maximizar a quantidade final de dinheiro no fluxo de caixa do investimento, expresso pela variável OR\$f. Variáveis e restrições são descritas nas Tabelas 3 e 4 . Os termos independentes estão descritos no Anexo IV.

Os vinte valores de preços semestrais do leite foram gerados aleatoriamente a partir de cinqüenta preços (pagos em $\mathrm{R} \$$ corrente aos produtores de leite de Goiás), média móvel a partir de preços mensais históricos (período de janeiro de 2002 a fevereiro de 2006) do litro de leite bovino (Anexos I e II). Para isso utilizouse procedimento de média (de preços de 6 meses) móvel e geração de números aleatórios disponíveis em planilha Excel.

3.2.1. Descrição de variáveis contempladas em cada período de tempo $j$

O modelo matemático incorpora nos anos de planejamento as variáveis descritas na Tabela 3. Note-se que o código de cada variável pode ser de até 8 caracteres quando se utiliza o formato Mathematical Programming System (MPS), preconizado pela IBM e apropriado como input para diferentes "solvers" ou programas computacionais.

3.2.2. Descrição das restrições para $o$ ano $j$ do modelo.

As restrições usadas no problema de programação linear são as descritas na Tabela 4.

3.2.3. Descrição dos coeficientes das variáveis do modelo.

O Anexo IV contém uma descrição dos coeficientes do modelo. 
Tabela 3: Descrição, numeração e codificação de variáveis para o ano $j$ do modelo.

\begin{tabular}{|c|c|c|}
\hline Descrição da variável & $\mathbf{N} .^{\circ}$ & Código \\
\hline Ano $j$ cultivo de milho área 1 silagem (ha) & 1 & $\mathrm{MiSCa1}_{j}$ \\
\hline Ano $j$ cultivo de cana área 2 para vaca cruzada (ha) & 2 & CaC_a2yj \\
\hline Ano $j$ cultivo de milheto área 1 pastagem vaca holandesa (ha) & 3 & Milyj $\mathrm{Hz}$ \\
\hline Ano j cultivo de milheto área 1 pastagem vaca cruzada (ha) & 4 & Milyj $_{j} \mathrm{Cz}$ \\
\hline Ano j pasto tanzânia rotacionado vaca holandesa (ha) & 5 & Taroy $_{j} \mathrm{~Hz}$ \\
\hline Ano $j$ pasto tanzânia rotacionado vaca cruzada (ha) & 6 & Taroy $_{j} C z$ \\
\hline Ano $j$ pasto Braquiária vaca cruzada (ha) & 7 & $p a 2 y_{j} B r$ \\
\hline Ano j orçamento de custos diretos de insumos (R\$) & 8 & Sinsy $_{j} 09$ \\
\hline Ano $j$ fazenda (recursos e despesas) (und) & 9 & $R e D \_F y_{j}$ \\
\hline Ano j mão-de-obra contratada eventual (diária) & 10 & MOC_ev \\
\hline Ano $j$ mão-de-obra contratada rotina (diária) & 11 & $M O C \_r o_{j}$ \\
\hline Ano $j$ aluguel de hora trator padrão (hr) & 12 & $\mathrm{TrHMy}_{j}$ \\
\hline Ano $j$ aluguel de hora trator com ensiladeira (hr) & 13 & $T r H M E y_{j}$ \\
\hline Ano $j$ estoque silagem milho disponível vaca holandesa (kg) & 14 & $E S M y_{j} H z$ \\
\hline Ano $j$ estoque silagem milho disponível vaca cruzada (kg) & 15 & $E S M y_{j} C z$ \\
\hline Ano j compra de silagem de milho para vaca holandesa $(\mathrm{kg})$ & 16 & $\mathrm{CSMHzy}_{j}$ \\
\hline Ano $j$ compra de silagem de milho para vaca cruzada $(\mathrm{kg})$ & 17 & $C S M C z y_{j}$ \\
\hline Ano $j$ vaca individual holandesa (und) & 18 & vacinH01 \\
\hline Ano $j$ produção de cria vaca holandesa (und) & 19 & Pr_criaH \\
\hline Ano $j$ vaca individual vaca cruzada (und) & 20 & vacinC01 \\
\hline Ano j produção de cria vaca cruzada (und) & 21 & Pr_criaC \\
\hline $\begin{array}{l}\text { Ano } j \text { amortização de investimento no rebanho vaca holandesa } \\
\text { (und) }\end{array}$ & 22 & AmInH01 \\
\hline $\begin{array}{l}\text { Ano j amortização de investimento no rebanho vaca cruzada } \\
\text { (und) }\end{array}$ & 23 & AmInC01 \\
\hline Ano j crédito custeio pecuário (R\$) & 24 & $C r C P y_{j}$ \\
\hline Ano j crédito custeio agrícola (R\$) & 25 & $\mathrm{CrCIy}_{j}$ \\
\hline Ano j crédito cédula do produtor rural (R\$) & 26 & $C r C P R y_{j}$ \\
\hline Ano $j$ custos de concentrado para vaca em lactação (R\$) & 27 & $C R \_V L y_{j}$ \\
\hline Ano j custos de produtos veterinários (R\$) & 28 & $C P V_{-} R y_{j}$ \\
\hline Ano $j$ custos de manutenção de pastagens (R\$) & 29 & $C M a n P y_{j}$ \\
\hline Ano $j$ custos de manutenção de equipamentos (R\$) & 30 & $C M a n E y_{j}$ \\
\hline Ano $j$ custos de manutenção de automóvel (R\$) & 31 & $C M a n C y_{j}$ \\
\hline Ano $j$ custos de sal mineral, e de inseminação (R\$) & 32 & CSAI_y \\
\hline Ano $j$ custos de manutenção da família (R\$) & 33 & $C M a n F y_{j}$ \\
\hline Ano j provisão de despesas de telefone $(\mathrm{R} \$)$ & 34 & $C R \_y_{j} 01$ \\
\hline Ano j provisão de despesas de energia elétrica $(\mathrm{R} \$)$ & 35 & $C R \_y_{j} 02$ \\
\hline Ano $j$ provisão de outras despesas de manutenção (R\$) & 36 & $C R \_y_{j} 03$ \\
\hline Ano j depreciação ou reserva de capital para repor ativos (R\$) & 37 & $C R \_y_{j} 04$ \\
\hline Ano $j$ venda de leite $1^{0}$ semestre $\left(\mathrm{l}\right.$ semestre ${ }^{-1}$ ) & 38 & jvenleia \\
\hline Ano $j$ venda de leite $2^{o}$ semestre (l semestre ${ }^{-1}$ ) & 39 & jvenleib \\
\hline $\begin{array}{l}\text { Ano } j \text { transferência carry-over silagem de milho para o ano } j+1 \\
(\mathrm{~kg})\end{array}$ & 40 & $T r \_E S M_{j+1}$ \\
\hline $\begin{array}{l}\text { Ano j transferência dinheiro do caixa jcap_\$a para jcap_\$b } \\
(\mathrm{R} \$)\end{array}$ & 41 & $j \operatorname{Tr} R \$ j b$ \\
\hline $\begin{array}{l}\text { Ano } j \text { transferência dinheiro do caixa (saldo positivo) } j b \text { para o } \\
(j+1) a(\mathrm{R} \$)\end{array}$ & 42 & jTrR\$jp \\
\hline Ano $j=n+1$ quantidade final de dinheiro no fluxo de caixa (R\$) & 43 & $O R \$ f$ \\
\hline
\end{tabular}


Tabela 4: Descrição, numeração e codificação das restrições para o ano $j$ do modelo.

\begin{tabular}{|c|c|c|}
\hline Descrição das restrições & N. ${ }^{\mathbf{o}}$ & Código \\
\hline Ano j Área abaixo da estrada lavoura1 (ha) & 1 & ja01lav1 \\
\hline Ano j Área abaixo da estrada safrinha (ha) & 2 & ja01saf1 \\
\hline Ano j Área com cana 01 (ha) & 3 & ja02can1 \\
\hline Ano j Pasto 01 Tanzânia rotacionado (ha) & 4 & ja03pa1 \\
\hline Ano j Pasto 02 Braquiaria brizantha (ha) & 5 & ja04pa2 \\
\hline Ano j Custo Total de Insumos (R\$) & 6 & jcdiLP \\
\hline Ano j Hora máquina trator aluguel padrão (und) & 7 & $j H M \_A P$ \\
\hline Ano j Hora máquina trator aluguel com ensiladeira (und) & 8 & jHM_Aens \\
\hline Ano j Mão-de-obra contratada (hr) & 9 & jMOCont \\
\hline Ano j Família residente com 1,5 mão-de-obra (hr) & 10 & Jfazenda \\
\hline Ano j Mão-de-obra família disponível (hr) & 11 & jMOFam \\
\hline Ano $j$ Estoque de silagem de milho $(\mathrm{kg})$ & 12 & jESMkg \\
\hline Ano $j$ Investimento no rebanho holandês (und) & 13 & jCap_IrH \\
\hline Ano $j$ Investimento no rebanho cruzado (und) & 14 & jCap_IrC \\
\hline Ano $j$ Cria produzida raça holandesa (und) & 15 & jCriaHz \\
\hline Ano $j$ Cria produzida raça cruzada (und) & 16 & jCriaCz \\
\hline Ano $j$ Consumo massa seca vaca holandesa semestre $a\left(\mathrm{~kg}\right.$ semestre $\left.^{-1}\right)$ & 17 & jCRmsHa \\
\hline Ano $j$ Consumo energia vaca holandesa semestre $a\left(\mathrm{~kg}\right.$ semestre $\left.{ }^{-1}\right)$ & 18 & jCRenHa \\
\hline Ano $j$ Consumo fibra vaca holandesa semestre $a\left(\mathrm{~kg}\right.$ semestre $\left.{ }^{-1}\right)$ & 19 & jCRfbHa \\
\hline Ano $j$ Consumo massa seca vaca holandesa semestre $b\left(\right.$ kg semestre $\left.^{-1}\right)$ & 20 & jCRmsHb \\
\hline Ano $j$ Consumo energia vaca holandesa semestre $b\left(\mathrm{~kg}\right.$ semestre $\left.{ }^{-1}\right)$ & 21 & jCRenHb \\
\hline Ano $j$ Consumo fibra vaca holandesa semestre $b\left(\mathrm{~kg}\right.$ semestre $\left.{ }^{-1}\right)$ & 22 & $j C R f b H b$ \\
\hline Ano $j$ Consumo massa seca vaca cruzada semestre $a\left(\mathrm{~kg}\right.$ semestre $\left.{ }^{-1}\right)$ & 23 & jCRmsCza \\
\hline Ano $j$ Consumo energia vaca cruzada semestre $a\left(\mathrm{~kg}\right.$ semestre $\left.{ }^{-1}\right)$ & 24 & jCRenCza \\
\hline Ano $j$ Consumo fibra vaca cruzada semestre $a\left(\mathrm{~kg}\right.$ semestre $\left.{ }^{-1}\right)$ & 25 & jCRfbCza \\
\hline Ano $j$ Consumo massa seca vaca cruzada semestre $b\left(\mathrm{~kg}\right.$ semestre $\left.{ }^{-1}\right)$ & 26 & jCRmsCzb \\
\hline Ano $j$ Consumo energia vaca cruzada semestre $b\left(\mathrm{~kg}\right.$ semestre $\left.{ }^{-1}\right)$ & 27 & jCRenCzb \\
\hline Ano $j$ Consumo fibra vaca cruzada semestre $b\left(\mathrm{~kg}\right.$ semestre $\left.{ }^{-1}\right)$ & 28 & $j C R f b C z b$ \\
\hline Ano $j$ Produção de leite semestre $a$ (litros) & 29 & jPley $_{j} a$ \\
\hline Ano $j$ Produção de leite semestre $b$ (litros) & 30 & jPley $_{j} b$ \\
\hline Ano j Consumo ração vacas em lactação (R\$) & 31 & $j R V L y_{j}$ \\
\hline Ano j Consumo de produtos veterinários (R\$) & 32 & jPVety ${ }_{j}$ \\
\hline Ano j Manutenção de pastagens (R\$) & 33 & $j M P a \_y_{j}$ \\
\hline Ano j Manutenção de equipamentos (R\$) & 34 & $j M E q \_y_{i}$ \\
\hline Ano j Manutenção de automóvel (R\$) & 35 & $j M C a_{-} y_{j}$ \\
\hline Ano j Sal mineral e inseminação (R\$) & 36 & jSinsy ${ }_{j}$ \\
\hline Ano j Consumo básico da família (R\$) & 37 & $j C F a \_y_{j}$ \\
\hline Ano $j$ Consumo de telefone $(\mathrm{R} \$)$ & 38 & jCte_y \\
\hline Ano $j$ Consumo de energia $(\mathrm{R} \$)$ & 39 & $j C E n \_y_{j}$ \\
\hline Ano j Outros custos (R\$) & 40 & $j O C_{-} y_{j}$ \\
\hline Ano $j$ Reserva de capital para repor ativos (R\$) & 41 & $j R C_{-} y_{j}$ \\
\hline Ano j Empréstimo crédito de custeio pecuário (R\$) & 42 & jECCPy \\
\hline Ano j Empréstimo crédito de custeio lavoura (R\$) & 43 & $j E C C L y_{j}$ \\
\hline Ano j Capital em caixa semestre a (R\$) & 44 & jcap_\$a \\
\hline Ano j Capital em caixa semestre b (R\$) & 45 & jcap_\$b \\
\hline Ano $n+1$ Caixa final $(\mathrm{R} \$)$ & 46 & Caixa $\$ f$ \\
\hline
\end{tabular}

Nota: Semestre $\boldsymbol{a}$ refere-se ao período chuvoso e semestre $\boldsymbol{b}$ período da seca. 


\subsection{Procedimento computacional para gerar soluções do modelo}

O procedimento de preparação do "Mathematical Programming System (MPS) input file" para o solver Lindo envolveu três etapas. A primeira foi 0 estabelecimento de todas as restrições compreendendo $\quad 0 \quad 1^{\circ}$ ano de planejamento com preparação de dados e especificação de seus parâmetros ou coeficientes. Para esta e as demais etapas de preparação do MPS input file usou-se o Office software da Empresa Microsoft. Optou-se pelo padrão MPS input file, introduzido inicialmente pela Empresa IBM, porque se trata de um padrão de arquivo de entrada para diferentes solvers, incluindo o Statistical Analysis System - Operational Research Procedure (SAS INSTITUTE, 2000). Em seguida, introduziu-se variáveis de transferência do primeiro para o segundo ano e utilizando-se de duas pastas do Excel foi possível especificar todo o modelo que compreende 416 restrições e 375 variáveis. O processo de geração do MPS input file, que é um arquivo do tipo texto ou ASC file, envolve a aplicação de uma macro incorporada no arquivo Excel.

\section{RESULTADOS}

Uma solução sintética do modelo é apresentada no Anexo V. Nele estão descritas todas as variáveis incluídas na solução, seguindo uma abordagem de fluxo de caixa periódico. Isto é, os valores de cada variável são dispostos em colunas para mostrar a dinâmica do negócio do primeiro ao décimo ano. Note-se que algumas variáveis do primeiro ano de planejamento não são incluídas nos anos seguintes.

Verifica-se que na solução os valores das quantidades de vacas holandesas 42,82 e de vacas cruzadas 42,96, deveriam ser números inteiros. Esses valores definem as quantidades de vacas do rebanho. Entretanto, nessa aplicação, as quantidades de vacas não foram definidas como variáveis inteiras porque essa solução específica não será utilizada para apoiar um processo de tomada de decisão de um determinado produtor. Ela visa demonstrar o potencial da ferramenta para avaliar política de crédito (6\% a.a.) rural. Para um aplicação visando estabelecer cenários para alternativas taxas de juros de crédito rural, o modelo deveria ser submetido a uma revisão pelos usuários ou formuladores de política.

Tanto vacas holandesas quanto cruzadas que foram adquiridas são vendidas após seis anos. Foram definidas no primeiro ano do plano de investimentos as quantidades de vacas. $\mathrm{Na}$ construção do modelo foram associados (seguindo o conceito de tabela price) às respectivas variáveis, o custo de aquisição e o preço de descarte de cada vaca. A atividade de produção de leite mostrou-se economicamente viável quanto à reposição de vacas.

Com um total de 86 vacas produzindo aproximadamente 1.000 litros/dia é possível assumir compromissos decorrentes de R\$100.000,00 (Tabela 2) de investimentos, financiados a uma taxa de juros de 6\%, e amortização em 30 anos, com um valor de sucata de R\$ $50.000,00$ no final do período. No fluxo de caixa estabelecido no modelo o valor da prestação (calculada pela Tabela price) de $\mathrm{R} \$ 6.257,12$ para amortizar o total de investimentos, é correspondente à "reserva capital para repor ativos" descrita na Tabela 1. Contudo, o valor de renda líquida no final do $20^{\circ}$ semestre do plano é de apenas R\$22.584,92. Assim, é importante considerar outras tecnologias que possam melhorar o desempenho econômico do sistema de produção.

\section{COMENTÁRIOS FINAIS}

O modelo apresenta a possibilidade de avaliar, em termos bioeconômicos, alternativas tecnológicas e estratégias de manejo para sistema de produção com atividade leiteira. 
A utilização extensiva de modelo como ferramenta efetiva de apoio à tomada de decisões dependerá dos interesses de responsáveis pela formulação, implementação, monitoramento, avaliação e revisão da política de crédito do PRONAF, que opera com uma taxa de juros inferior à taxa de $6 \%$ a.a., considerada no modelo.

O resultado importante desse estudo é a demonstração de que com as práticas identificadas (e consideradas) em um sistema (fazenda) de produção do mundo real e a taxa de juros de $6 \%$ ao ano para novos investimentos, superior às taxas estabelecidas pelo PRONAF (de 1 a $4 \%$ a.a. $)^{8}$, o negócio dinâmico de uma fazenda como aqui representado no modelo é economicamente sustentável (FERREIRA, 2007).

As tecnologias de pastagem irrigada, de recuperação de pastagem braquiária degradada e outras devem ser consideradas no caso de uso desse modelo como instrumento de apoio à uma revisão do Programa PRONAF. Isto implicaria em sua expansão, mas trata-se de uma detalhe relativamente simples. $\mathrm{O}$ importante é que o modelo está disponível para extensionistas, técnicos do PRONAF ou qualquer pessoa interessada na questão de crescimento de uma pequena fazenda orientada para produção de leite na região do Cerrado.

Com a elevação recente dos preços do leite, tanto nos mercados interno e externo, o cenário de médio prazo indica que os produtores estarão mais propensos a adoção de novas tecnologias (TONINI et al., 2007).

Entretanto, uma análise de sensibilidade do modelo considerando mudanças de taxas de juros e de tecnologias indicadas para sistemas de produção, que incorporam a atividade leiteira como sendo componente crucial do negócio, precisa ser requerida por analistas (usuários) do mundo real que

\footnotetext{
${ }^{8}$ O PRONAF tem estabelecido uma taxa efetiva de juros de $4 \%$ ao ano, com bônus de adimplência de $25 \%$. Isto, na prática, reduz a taxa de juros para 3\%, sempre que a parcela for paga até o dia do vencimento (PROGRAMA NACIONAL DE FORTALECIMENTO DA AGRICULTURA FAMILIAR, 2007).
}

ENGEVISTA, v. 9, n. 1, p. 57-81, junho 2007 tenham estabelecido suas perguntas passíveis de serem tratadas pelo modelo.

Muitos aspectos incorporados nesse modelo serão adotados em modelos mais amplos visando representar, por exemplo, um sistema de produção agrossilvipastoril enfatizando os aspectos de economia de escala e de escopo. As pequenas propriedades rurais precisam considerar a visão de economia de escopo porque a produção de apenas um ou dois produtos torna o negócio extremamente vulnerável em períodos de baixos preços de produtos recebidos pelos produtores.

\section{REFERÊNCIAS}

BAUMOL, W.T.; PANZAR, J.C.; WILLIG, R.D. Contestable markets and theory of industry structure. New York: Hardcourt Brace Javanovich, 1988. 538p.

BERGER, T.; SCHREINEMACHERS, P.; WOELCKE, J. Multi-agent simulation for the targeting of development policies in less-favored areas. Agricultural Systems, v. 88, p. 28-43, 2006.

BÖRNER, J.C. A bio-economic model of small-scale farmers' land use decisions and technology choice in the eastern Brazilian Amazon. PhD Thesis, Institut fur Landwirtschafliche Betriebslehre, Hamburg, 2005.

DENT, J.B.; HARRISON, S.H.; WOODFORD, K.B. Farm planning with linear programming: concept and practice. Sydney: Butterworths, 1986.

HARDAKER, J. B. A review of some farm management research methods for small-farm development in LDCs. Journal of Agricultural Economics, v. 30, p. 315-323, 1979
FERREIRA, C.R.C.O. Como o agricultor familiar pode conseguir e manter o financiamento rural e como se dá à relação com os bancos. Brasília: Ministério do Desenvolvimento Agrário, Secretaria de Agricultura Familiar, 2007. Disponível em: <http://www.mda.gov.br/saf/arquivos/11 37912740.doc>. Acesso em: 3 out. 2007. 
HAZELL, P.; Norton, R. Mathematical Programming for Economic Analysis in Agriculture. New York: Macmillan, 1986.

IBSNAT PROJECT. A Prototype Activity to Develop a Decision Support System for Overcoming Bottlenecks in Agro-production Technology in the Tropical and Subtropical Regions. Phase II, Project No. 936-4054, p. 64, 1987.

JANSSEN, W.; GOLDSWORTHY, P. Multidisciplinary research for natural resource management: conceptual and practical implications. Agricultural Systems, v. 51, p. 259-279, 1996.

KUYVENHOVEN, A. Creating an enabling environment: policy conditions for less-favored areas. Food Policy, v. 10, n. 4, p. 407-429, 2004.

MANKIW, G. Introdução à Economia.

2. ed. Rio de Janeiro: Campus, 2001.

MAXWELL, S. The role of case studies in farming systems research. Agricultural Administration, v. 21, p. 147-180, 1986.

OUDE LAUSINK, A.; STEFANOU, S.E. Dynamic area allocation and economics of scale and scope. Journal of Agricultural Economics, v. 52, p. 38-52, 2001.

PATRICK, G.F.; EISGRUBER, L.M. The impact of managerial ability and capital structure on growth of the farm firm. American Journal of Agricultural Economics, v. 50, n. 3, p. 492-506, 1968.

PROGRAMA NACIONAL DE FORTALECIMENTO DA AGRICULTURA FAMILIAR. Disponível em: $<$ http://www.ematerce.ce.gov.br/pronaf.h tm>. Acesso em: 3 out. 2007.

RUBEN, R., PENDER, J. Rural diversity and heterogeneity in less-favoured areas: the quest for policy targeting. Food Policy, v. 19, n. 4, p. 303-320, 2004.

SAS INSTITUTE: PROC LP: Converting MPS Format. Disponível in: $<$ http://www.sas.com/mbs $>$. Acesso em: 5 jul. 2000.
TONINI, M.G.; ROSA, F.R.T.; TORRES JR., A.M. Pecuária: cresce o confinamento. Agroanalysis, v. 27, n. 8, p. 27-28, 2007.

VELOSO, R.F.; FERNANDES, F.B.; BARIONI, L.G.; CHAIB FILHO, H.; SILVA, A.P.; COLOCA, A.A. Tomada de decisões gerenciais no âmbito de duas fazendas familiares no sul goiano. Cadernos de Ciência \& Tecnologia, v. 20, n. 2, p. 305-323, 2003.

VIDAL, R.V.V. Operational research: a multidisciplinary field. Pesquisa Operacional, v. 26, n. 1, p. 69-90, 2006.

VILELA， L.; BARCELLOS, A.O.; SOUSA, D.M.G. Benefícios da integração entre lavoura e pecuária. Planaltina: Embrapa Cerrados, 21 p. (Embrapa Cerrados. Documentos, 42), 2001. 


\section{ANEXO I}

Dados (usados para uma rodada do modelo) de preços semestrais (gerados aleatoriamente a partir de uma série de preços de média -de 6 meses- móvel) do litro (em R\$) de leite pagos aos produtores de Goiás.

\begin{tabular}{|c|c|c|c|c|c|c|c|c|c|c|}
\hline Semestre & 1 & 2 & 3 & 4 & 5 & 6 & 7 & 8 & 9 & 10 \\
\hline (R\$/litro) & 0,49 & 0,42 & 0,47 & 0,30 & 0,27 & 0,45 & 0,44 & 0,44 & 0,40 & 0,40 \\
\hline
\end{tabular}

\begin{tabular}{|c|c|c|c|c|c|c|c|c|c|c|}
\hline Semestre & 11 & 12 & 13 & 14 & 15 & 16 & 17 & 18 & 19 & 20 \\
\hline (R\$/litro) & 0,42 & 0,36 & 0,39 & 0,48 & 0,27 & 0,40 & 0,27 & 0,42 & 0,49 & 0,49 \\
\hline
\end{tabular}

Séries de preços $(\mathrm{R} \$ / \mathrm{l})$ mensal e semestral estimado do litro de leite em Goiás.

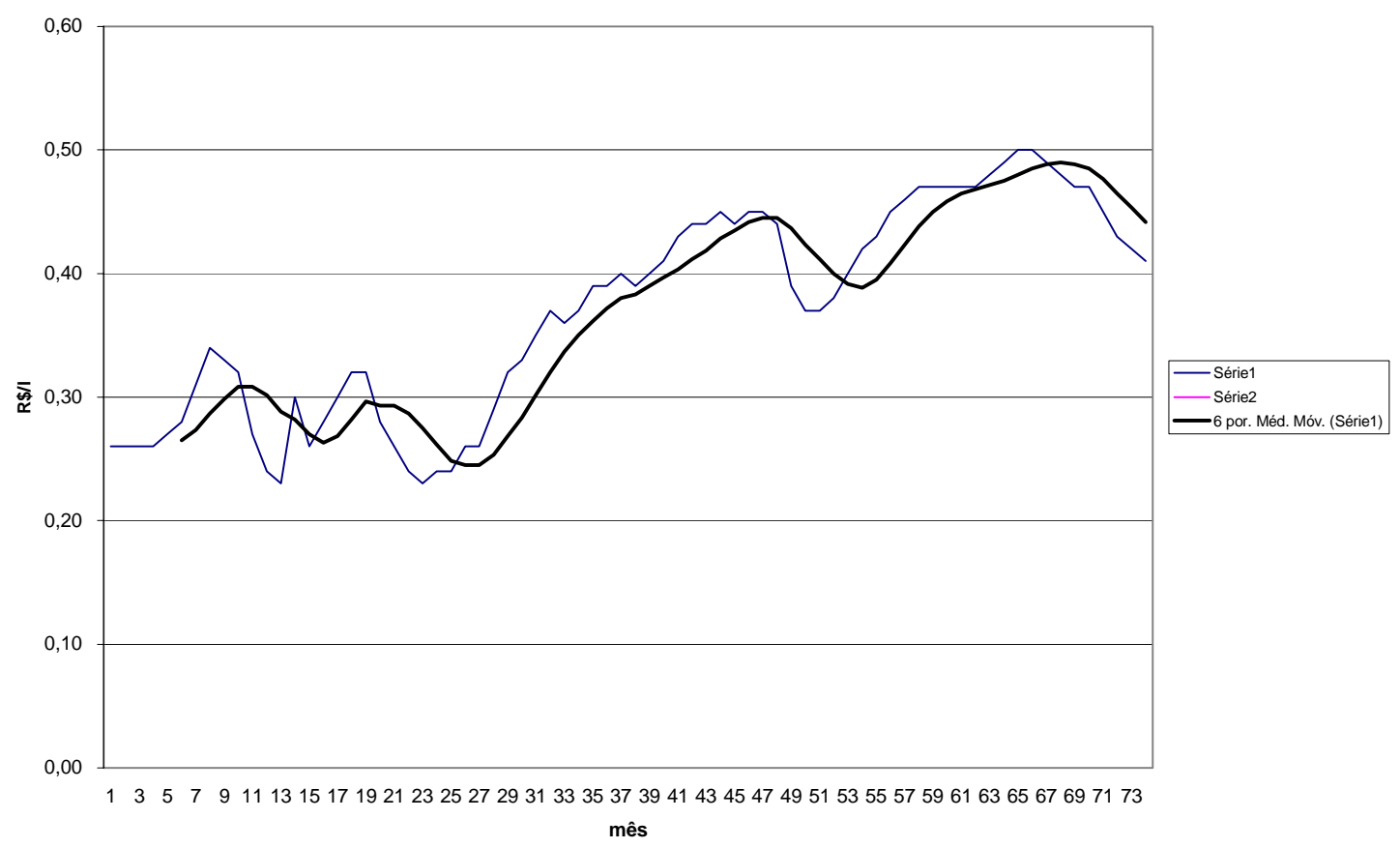




\section{ANEXO II}

Preço (pago aos produtores em R\$) do litro de leite em Goiás.

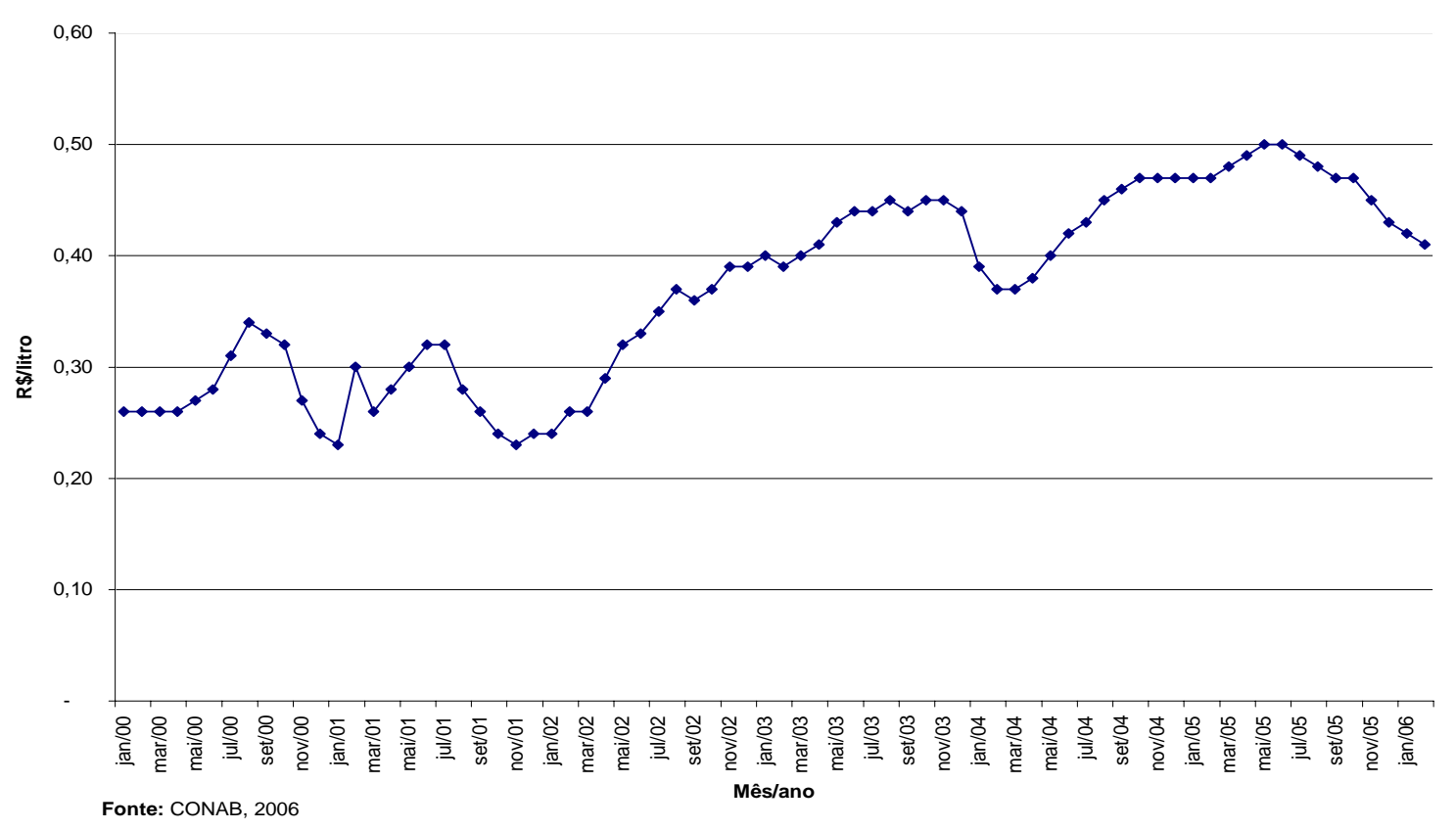

Preço (pago aos produtores em US\$ corrente) do litro de leite em Goiás.

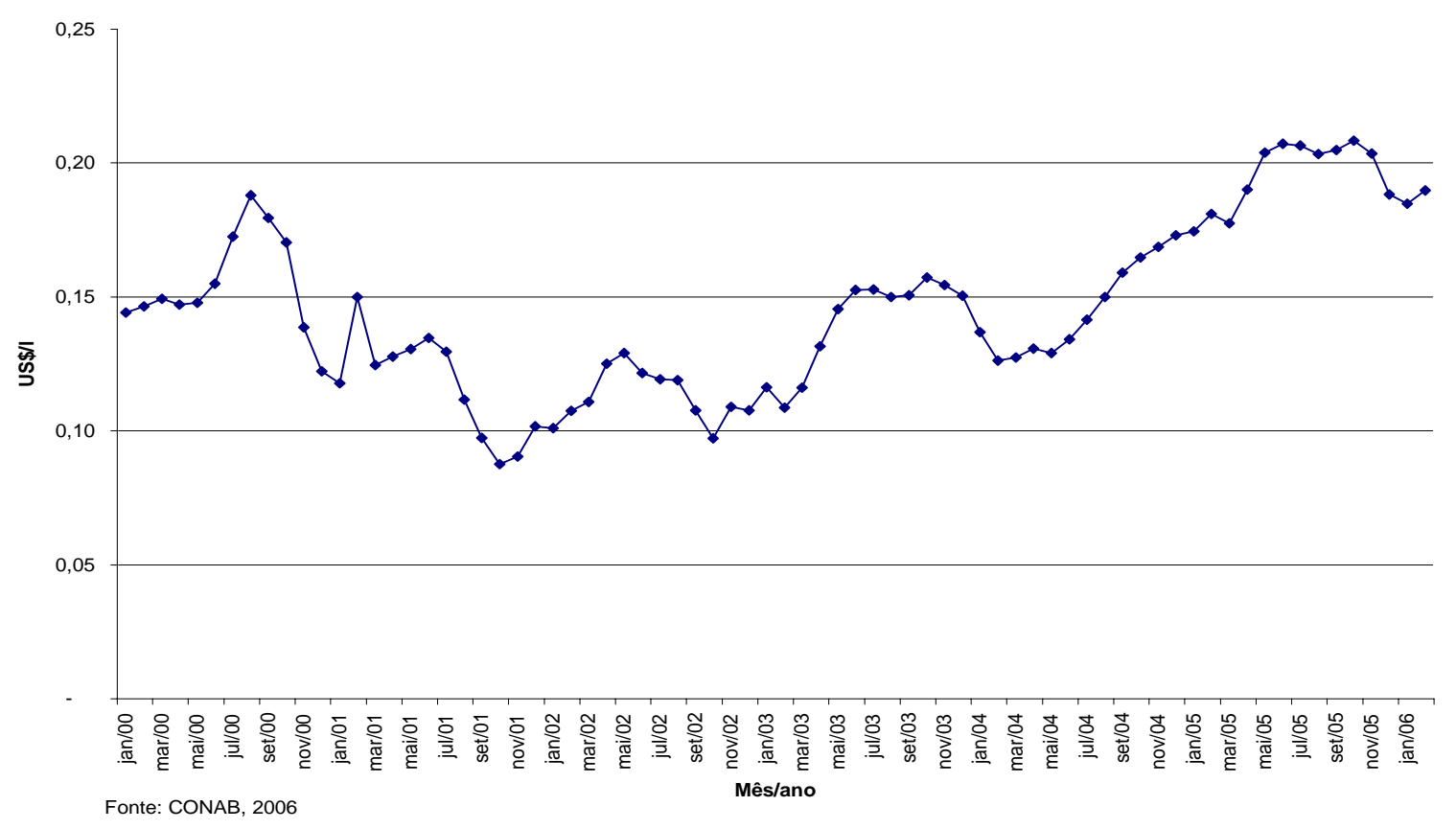




\section{ANEXO III}

Formulação matemática

\section{Max $O R \$ f$}

sujeito a

$$
\begin{aligned}
& \text { ja01lav1) }\left\{\begin{array}{l}
{\operatorname{MisCa} 1 y_{j} \leq \text { área }_{\text {Mis }}, j=1} \text { MiSCa1 }_{j}+\text { MiSCa1 }_{j+1} \leq 0, j=1 \ldots n-1
\end{array}\right. \\
& \text { ja01saf } 1)\left\{- \text { MiSCa1 }_{j}+\text { mily }_{j} \mathrm{~Hz}+\text { mily }_{j} \mathrm{Cz} \leq 0, j=1 \ldots n\right. \\
& \text { ja02can1) }\left\{\begin{array}{l}
\text { CaC_a } 2 y_{j} \leq \text { área }_{C a}, j=1 \\
-C a C_{-} a 2 y_{j}+C a C_{-} a 2 y_{j+1} \leq 0, j=1 \ldots n-1
\end{array}\right. \\
& \text { ja03pa1) }\left\{\begin{array}{l}
\text { Taroy }_{j} \mathrm{~Hz}+\text { Taroy }_{j} \mathrm{Cz} \leq \text { área }_{\mathrm{Tz}}, j=1 \\
- \text { Taroy }_{j} \mathrm{~Hz}-\text { Taroy }_{j} \mathrm{Cz}+\text { Taroy }_{j+1} \mathrm{~Hz}+\text { Taroy }_{j+1} \mathrm{Cz} \leq 0, j=1 \ldots . . n-1
\end{array}\right. \\
& \text { ja04lpa2) }\left\{\begin{array}{l}
{\text { pa } 2 y_{j} \mathrm{Br} \leq \text { área }_{\mathrm{Br}}, j=1}^{-\mathrm{pa}_{2} \mathrm{y}_{j} \mathrm{Br}+\mathrm{pa}_{2} \mathrm{y}_{j+1} \mathrm{Br} \leq 0, j=1 . . . n-1}
\end{array}\right.
\end{aligned}
$$

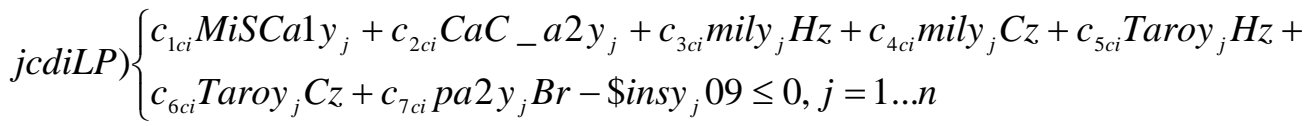$$
j H M_{2} \text { AP) }\left\{C_{\text {нмР }} \text { MiSCa1 }_{j}-\operatorname{TrHMy}_{j} \leq 0, j=1 \ldots n\right.
$$$$
\left.j H M_{-} \text {Aens }\right)\left\{c_{\text {HMEnS }} \text { MiSCa1 }_{j}-\text { TrHMEy }_{j} \leq 0, j=1 \ldots n\right.
$$

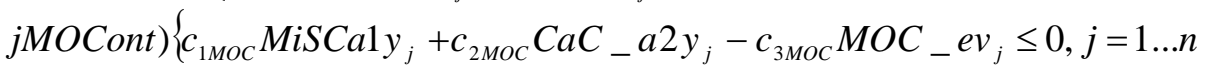$$
\text { jfazenda) }\left\{\begin{array}{l}
\operatorname{Re} D_{-} F y_{j}=1, j=1 \\
-\operatorname{Re} D_{-} F y_{j}+\operatorname{Re} D_{-} F y_{j+1}=0, j=1 \ldots n-1
\end{array}\right.
$$$$
\text { jMOFam })\left\{\begin{array}{l}
c_{1 M O F} \text { vacinH } 01+c_{2 M O F} \text { Pr_criaH }+c_{3 M O F} \text { vacinC } 01+c_{4 M O F} \text { Pr_criaC }+ \\
c_{5 M O F} \text { MiSCay }_{j}+c_{6 M O F} C a C_{-} a 2 y_{j}+c_{7 M O F} \text { mily }_{j} H z+c_{8 M O F} \text { mily }_{j} C z- \\
c_{9 M O F} \operatorname{Re} D_{-} \text {Fy }_{j}-c_{10 M O F} M O C_{-} r_{j}+c_{11 M O F} C S M H z y_{j}+c_{12 M O F} C S M C z y_{j} \leq 0, j=1 \ldots n
\end{array}\right.
$$$$
j E S M k g)\left\{\begin{array}{l}
-c_{1 E S} T r_{-} E S M_{j}-c_{2 E S} \operatorname{MiSCa}_{j}+\operatorname{ESMy}_{j} \mathrm{~Hz}+\mathrm{ESMy}_{j} \mathrm{Cz}+\operatorname{Tr} r_{-} E S M_{j+1} \leq 0, j=1 \ldots n \\
T R_{-} E S M_{j}=0, j=1, n+1
\end{array}\right.
$$

jCap_IrH $\{$ vacinH $01-\mathrm{AmInH} 01 \leq 0, j=1$

jCap_IrC $\{$ vacinC01-AmInC01 $\leq 0, j=1$

jCriaHz $\{$ vacinH $01-$ Pr_CriaH $=0, j=1$

jCriaCz $\{$ vacinC01-Pr_CriaC $=0, j=1$

jCRmsHa $)\left\{c_{1 \mathrm{Hms}-\mathrm{a}}\right.$ vacinH $01-c_{2 \mathrm{Hms}-a}$ mily $_{j} \mathrm{~Hz}-\mathrm{c}_{3 \mathrm{Hms}-\mathrm{a}}$ Taroy $_{j} \mathrm{~Hz}-\mathrm{c}_{4 \mathrm{Hms}-a} \mathrm{CSMHzy}_{j} \leq 0, j=1 . . . n$

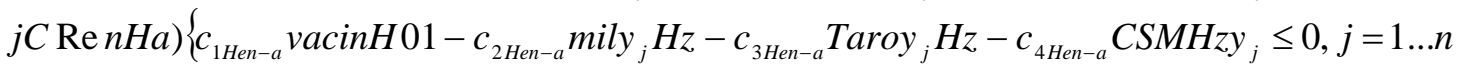

$j C R f b H a)\left\{c_{1 H f b-a}\right.$ vacinH $01-c_{2 H f b-a}$ mily $_{j} H z-c_{3 H f b-a}$ Taroy $_{j} H z-c_{4 H f b-a} C$ SMHzy $_{j} \leq 0, j=1 \ldots n$

$j \mathrm{CRmsHb})\left\{c_{1 \mathrm{Hms}-\mathrm{b}}\right.$ vacinH $01-c_{2 \mathrm{Hms}-b}$ Taroy $_{j} \mathrm{~Hz}-c_{3 \mathrm{Hms}-b} \mathrm{ESMy}_{j} \mathrm{~Hz} \leq 0, j=1 \ldots n$

$j C \operatorname{Re} n H b)\left\{c_{1 H e n-b}\right.$ vacinH $01-c_{2 \text { Hen-b }}$ Taroy $_{j} H z-c_{3 H e n-b} E S M y_{j} H z \leq 0, j=1 \ldots n$

$j C R m s C z a)\left\{c_{1 C m s-a}\right.$ vacinC01- $c_{2 C m s-a}$ mily $_{j} C z-c_{3 C m s-a}$ Taroy $_{j} C z-c_{4 C m s-a} p a 2 y_{j} B r \leq 0, j=1 \ldots n$

$j C$ Re $n C z a)\left\{c_{1 C e n-a}\right.$ vacinC01- $c_{2 C e n-a}$ mily $_{j} C z-c_{3 C e n-a}$ Taroy $_{j} C z-c_{4 C e n-a} p a 2 y_{j} B r \leq 0, j=1 \ldots n$

$j C$ Re $n C z a)\left\{c_{1 C f b-a}\right.$ vacinC01- $c_{2 C f b-a}$ mily $_{j} C z-c_{3 C f b-a}$ Taroy $_{j} C z-c_{4 C f b-a}$ pa $2 y_{j} B r \leq 0, j=1 \ldots n$

$j C R f b H b)\left\{c_{1 H f b-b}\right.$ vacinH $01-c_{2 H f b-b}$ Taroy $_{j} H z-C_{3 H f b-b} E$ SMy $_{j} \mathrm{~Hz} \leq 0, j=1 \ldots . n$ 


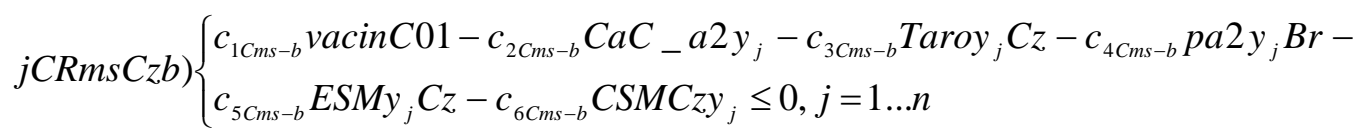
$j C \operatorname{Re} n C z b)\left\{\begin{array}{l}c_{1 C e n-b} \text { VacinC01- } c_{2 C e n-b} C a C_{-} a 2 y_{j}-c_{3 C e n-b} \text { Taroy }_{j} C z-C_{4 C e n-b} p a 2 y_{j} B r- \\ c_{5 C e n-b} E S M y_{j} C z-c_{6 C e n-b} C S M C z y_{j} \leq 0, j=1 \ldots n\end{array}\right.$

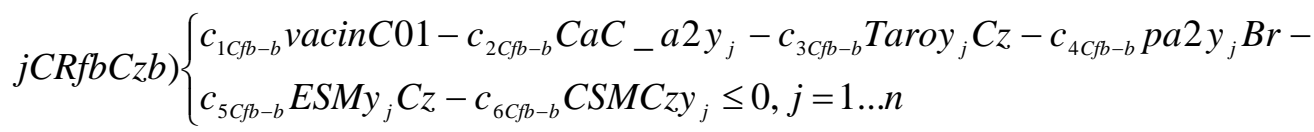
jPley $\left._{j} a\right)\left\{-c_{1 P l e}\right.$ vacinH $01-c_{2 P l e}$ vacinC $01+$ jvenleia $\leq 0, j=1 \ldots n$ jPley $\left.{ }_{j} b\right)\left\{-c_{1 P l e}\right.$ vacinH $01-c_{2 P l e}$ vacinC $01+$ jvenleib $\leq 0, j=1 \ldots n$ $\left.j R V L y_{j}\right)\left\{c_{1 R V L}\right.$ VacinH $01+c_{2 R V L}$ VacinC01-CR_VLy $y_{j} \leq 0, j=1 \ldots n$ $j P$ Vety $\left._{j}\right)\left\{c_{1 P V e t}{\text { vacinH } 01+C_{2 P V e t}}_{\text {vacinC0 }}-C P V_{-} R y_{j} \leq 0, j=1 \ldots n\right.$ $\left.j M P a_{-} y_{j}\right)\left\{c_{1 M P a}\right.$ vacinH $01+c_{2 M P a}$ vacinC01-CManPy ${ }_{j} \leq 0, j=1 . . . n$ $\left.j M E q_{-} y_{j}\right)\left\{c_{M E q} \operatorname{Re} D_{-} F y_{j}-C M a n E y_{j} \leq 0, j=1 \ldots n\right.$ $\left.j M C a_{-} y_{j}\right)\left\{c_{M C a} \operatorname{Re} D_{-} F y_{j}-C M a n C y_{j} \leq 0, j=1 \ldots n\right.$ $j$ Sinsy $\left._{j}\right)\left\{c_{1 S A I}\right.$ vacinH $01+c_{2 S A I}$ vacinC01-CSAI $y_{j} \leq 0, j=1 \ldots n$ $\left.j C F a_{-} y_{j}\right)\left\{c_{\text {ManFa }} \operatorname{Re} D_{-} F y_{j}-C_{M a n F y} \leq 0, j=1 \ldots n\right.$ jCte $\left.y_{j}\right)\left\{c_{C t e} \operatorname{Re} D_{-} F y_{j}-C R_{-} y_{j} 01 \leq 0, j=1 . . . n\right.$ $\left.j C E n_{-} y_{j}\right)\left\{c_{C e n} \operatorname{Re} D_{-} F y_{j}-C R_{-} y_{j} 02 \leq 0, j=1 \ldots . n\right.$ $\left.j O C_{-} y_{j}\right)\left\{c_{O C} \operatorname{Re} D_{-} F y_{j}-C R_{-} y_{j} 03 \leq 0, j=1 \ldots n\right.$ $\left.j R C_{-} y_{j}\right)\left\{c_{R C} \operatorname{Re} D_{-} F y_{j}-C R_{-} y_{j} 04 \leq 0, j=1 . . . n\right.$ $\left.j E C C P y_{j}\right)\left\{C r C P y_{j} \leq V E C C P, j=1 . . . n\right.$ $\left.j E C C L y_{j}\right)\left\{\right.$ CrCIy $_{j} \leq$ VECCL,$j=1 . . . n$

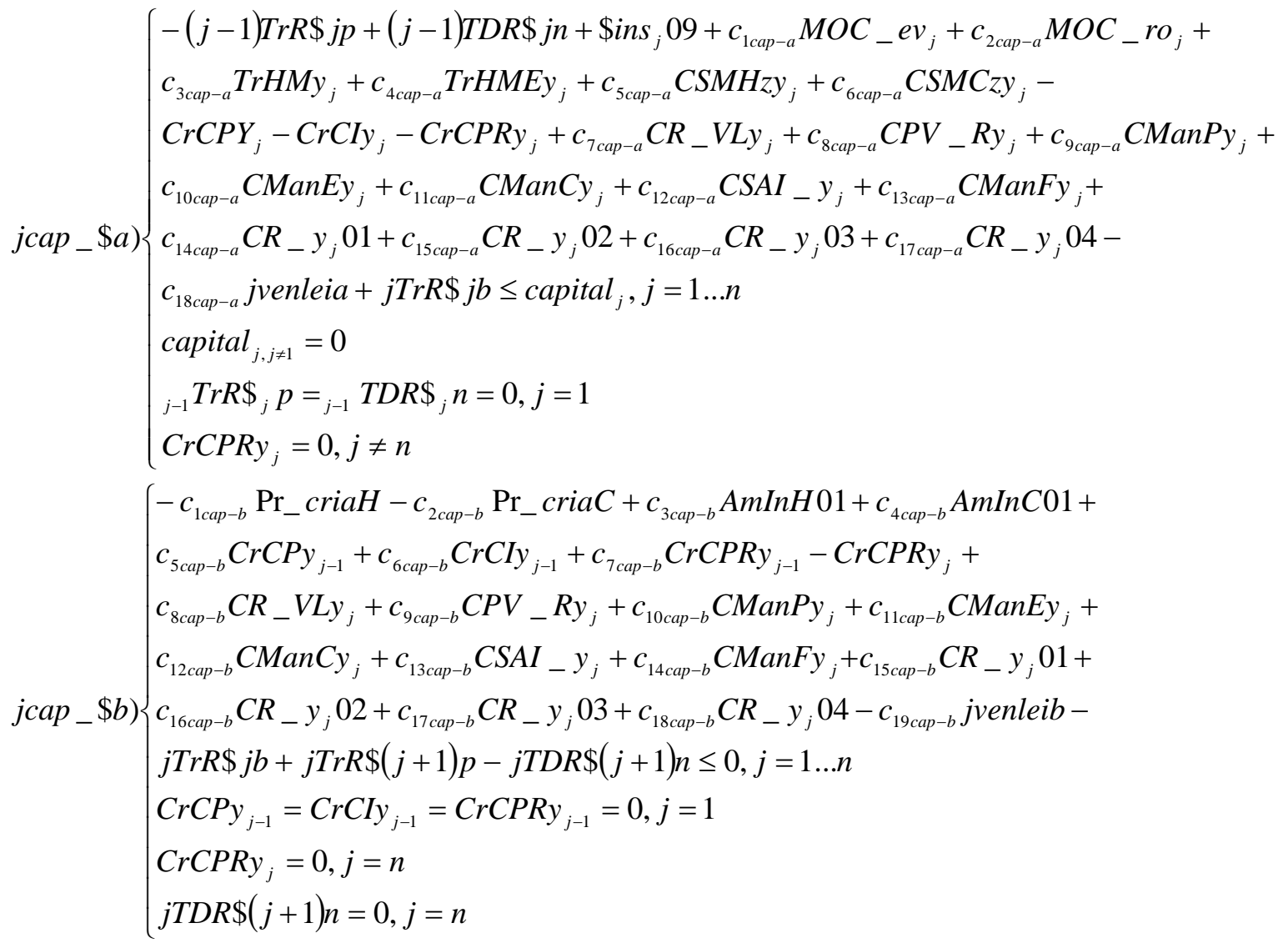

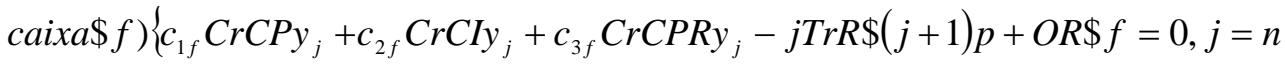


Descrição, numeração e codificação dos coeficientes do modelo.

\begin{tabular}{|c|c|c|c|}
\hline Descrição & $\mathbf{N .}^{\circ}$ & Und & Código \\
\hline Valor dos custos diretos para cultivo de 1 ha de milho $\mathrm{p} /$ silagem & 1 & $\mathrm{R} \$$ & $c_{1 c i}$ \\
\hline Valor dos custos diretos para cultivo de 1 ha de cana & 2 & $\mathrm{R} \$$ & $c_{2 c i}$ \\
\hline Valor dos custos diretos $\mathrm{p} /$ cultivo de 1 ha de milheto safrinha $\mathrm{Hz}$ & 3 & $\mathrm{R} \$$ & $c_{3 c i}$ \\
\hline Valor dos custos diretos $\mathrm{p} /$ cultivo de 1 ha de milheto safrinha $\mathrm{Cz}$ & 4 & $\mathrm{R} \$$ & $C_{4 c i}$ \\
\hline Valor dos custos diretos p/ cultivo de 1 ha de Tanzânia Hz & 5 & $\mathrm{R} \$$ & $C_{5 c i}$ \\
\hline Valor dos custos diretos p/ cultivo de 1 ha de Tanzânia $\mathrm{Cz}$ & 6 & $\mathrm{R} \$$ & $c_{6 c i}$ \\
\hline Valor dos custos diretos p/ cultivo de 1 ha de Braquiária $\mathrm{Cz}$ & 7 & $\mathrm{R} \$$ & $c_{7 c i}$ \\
\hline $\mathrm{N}^{\circ}$ de horas requeridas de trator padrão com grade $\mathrm{p} /$ cultivo milho & 8 & Hs & $c_{H M P}$ \\
\hline $\mathrm{N}^{\circ}$ de horas requeridas de trator com ensiladeira p/ cultivo milho & 9 & Hs & $C_{\text {HMEns }}$ \\
\hline Mão-de-obra (em horas) requerida para cultivo de milho silagem & 10 & Hs & $C_{1 M O C}$ \\
\hline Mão-de-obra (em horas) requerida para cultivo de cana & 11 & Hs & $C_{2 M O C}$ \\
\hline Mão-de-obra (em horas) contratada & 12 & Hs & $c_{3 M O C}$ \\
\hline Mão-de-obra (em horas) familiar p/ cultivo de milho silagem & 13 & Hs & $C_{1 M O F}$ \\
\hline Mão-de-obra (em horas) familiar p/ colheita e proc. de cana & 14 & Hs & $C_{2 M O F}$ \\
\hline Mão-de-obra (em horas) familiar p/ cultivo de milheto $\mathrm{Hz}$ & 15 & Hs & $C_{3 M O F}$ \\
\hline Mão-de-obra (em horas) familiar p/ cultivo de milheto $\mathrm{Cz}$ & 16 & Hs & $C_{4 M O F}$ \\
\hline Mão-de-obra (em horas) familiar ofertada durante 1 ano & 17 & Hs & $C_{5 M O F}$ \\
\hline Mão-de-obra (em horas) contratada para complementar MOF & 18 & Hs & $C_{6 M O F}$ \\
\hline Mão-de-obra (em horas) requerida p/ manejo de sil. Adquirida $\mathrm{Hz}$ & 19 & Hs & $C_{7 M O F}$ \\
\hline Mão-de-obra (em horas) requerida p/ manejo de sil. Adquirida Cz & 20 & Hs & $C_{8 M O F}$ \\
\hline Mão-de-obra (em horas) requerida por vaca $\mathrm{Hz}$ & 21 & Hs & $C_{9 M O F}$ \\
\hline Mão-de-obra (em horas) requerida por cria $\mathrm{Hz}$ & 22 & Hs & $C_{10 M O F}$ \\
\hline Mão-de-obra (em horas) requerida por vaca $\mathrm{Cz}$ & 23 & Hs & $c_{11 \mathrm{MOF}}$ \\
\hline Mão-de-obra (em horas) requerida por cria $\mathrm{Cz}$ & 24 & Hs & $C_{12 M O F}$ \\
\hline Transferência de $1 \mathrm{~kg}$ (com 20\% de perdas) de silagem p/ an & 25 & $\mathrm{Kg}$ & $c_{1 E S}$ \\
\hline Produtividade (em kg) de silagem de milho por ha & 26 & $\mathrm{Kg}$ & $C_{2 E S}$ \\
\hline Consumo (em kg) semestral de matéria seca (ms) por vaca Hz (nas águas) & 27 & $\mathrm{Kg}$ & $C_{1 H m s-a}$ \\
\hline Produtividade (em kg) de ms de milheto por ha p/ vaca Hz (nas águas) & 28 & $\mathrm{Kg}$ & $C_{2 H m s-a}$ \\
\hline Produtividade (em kg) de ms de Tanzânia por ha p/ vaca Hz (nas águas) & 29 & $\mathrm{Kg}$ & $C_{3 H m s-a}$ \\
\hline Quantidade (em kg) de ms/kg de silagem comprada p/ vaca Hz (nas águas) & 30 & $\mathrm{Kg}$ & $C_{4 H m s-a}$ \\
\hline Consumo (em kcal) semestral de energia (en) por vaca Hz (nas águas) & 31 & kcal & $c_{1 \mathrm{Hen}-\mathrm{a}}$ \\
\hline Produtividade (em kcal) de en de milheto por ha p/ vaca $\mathrm{Hz}$ (nas águas) & 32 & kcal & $C_{2 H e n-a}$ \\
\hline Produtividade (em kcal) de en de Tanzânia por ha p/ vaca Hz (nas águas) & 33 & kcal & $C_{3 H e n-a}$ \\
\hline Quantidade (em kcal) de en/kg de silagem comprada p/ vaca Hz (nas águas) & 34 & kcal & $C_{4 H e n-a}$ \\
\hline Consumo (em kg) semestral de fibra (fb) por vaca Hz (nas águas) & 35 & $\mathrm{Kg}$ & $c_{1 H f b-a}$ \\
\hline Produtividade (em kg) de fb de milheto por ha p/ vaca $\mathrm{Hz}$ (nas águas) & 36 & $\mathrm{Kg}$ & $C_{2 H}$ \\
\hline Produtividade (em kg) de fb de Tanzânia por ha p/ vaca Hz (nas águas) & 37 & $\mathrm{Kg}$ & $C_{3 H f b-a}$ \\
\hline Quantidade (em kg) de fb/kg de silagem comprada p/ vaca $\mathrm{Hz}$ (nas águas) & 38 & $\mathrm{Kg}$ & $C_{4 H f b-a}$ \\
\hline Consumo (em kg) semestral de matéria seca (ms) por vaca Hz (na seca) & 39 & $\mathrm{Kg}$ & $c_{1 \text { Hms-b }}$ \\
\hline Produtividade (em kg) de ms de Tanzânia por ha p/ vaca Hz (na seca) & 40 & $\mathrm{Kg}$ & $C_{2 H m s-b}$ \\
\hline Quantidade (em kg) de ms/kg de silagem comprada p/ vaca Hz (na seca) & 41 & $\mathrm{Kg}$ & $c_{3 H_{i}}$ \\
\hline Consumo (em kcal) semestral de energia (en) por vaca Hz (na seca) & 42 & Kcal & $c_{1 \mathrm{Hen}-\mathrm{b}}$ \\
\hline Produtividade (em kcal) de en de Tanzânia por ha p/ vaca $\mathrm{Hz}$ (na seca) & 43 & Kcal & $C_{2 H e n-b}$ \\
\hline Quantidade (em kcal) de en/kg de silagem comprada p/ vaca Hz (na seca) & 44 & Kcal & $C_{3 H e n-b}$ \\
\hline Consumo (em kg) semestral de fibra (fb) por vaca $\mathrm{Hz}$ (na seca) & 45 & $\mathrm{Kg}$ & $c_{1 H f b-b}$ \\
\hline Produtividade (em kg) de fb de Tanzânia por ha p/ vaca Hz (na seca) & 46 & $\mathrm{Kg}$ & $C_{2 H f b-b}$ \\
\hline Quantidade (em kg) de fb/kg de silagem comprada p/ vaca Hz (na seca) & 47 & $\mathrm{Kg}$ & $C_{3 H f b-b}$ \\
\hline Consumo (em kg) semestral de matéria seca (ms) por vaca Cz (nas águas) & 48 & $\mathrm{Kg}$ & $C_{1 \text { Cms-a }}$ \\
\hline Produtividade (em kg) de ms de milheto por ha p/ vaca Cz (nas águas) & 49 & $\mathrm{Kg}$ & $C_{2 C m s-a}$ \\
\hline Produtividade (em kg) de ms de Tanzânia por ha p/ vaca Cz (nas águas) & 50 & $\mathrm{Kg}$ & $C_{3 \text { Cms-a }}$ \\
\hline Produtividade (em kg) de ms de Braquiária por ha p/ vaca Cz (nas águas) & 51 & $\mathrm{Kg}$ & $C_{4 C m s-a}$ \\
\hline
\end{tabular}

ENGEVISTA, v. 9, n. 1, p. 57-81, junho 2007 


\begin{tabular}{|c|c|c|c|}
\hline Consumo (em kcal) semestral de energia (en) por vaca Cz (nas águas) & 52 & Kcal & $c_{1 C e n-a}$ \\
\hline Produtividade (em kcal) de en de milheto por ha p/ vaca Cz (nas águas) & 53 & Kcal & $C_{2 C e n-a}$ \\
\hline Produtividade (em kcal) de en de Tanzânia por ha p/ vaca Cz (nas águas) & 54 & Kcal & $C_{3 C e n-a}$ \\
\hline Produtividade (em kcal) de en de Braquiária por ha p/ vaca Cz (nas águas) & 55 & Kcal & $C_{4 C e n-a}$ \\
\hline Consumo (em kg) semestral de fibra (fb) por vaca Cz (nas águas) & 56 & $\mathrm{Kg}$ & $c_{1 C f b-a}$ \\
\hline Produtividade (em kg) de fb de milheto por ha p/ vaca Cz (nas águas) & 57 & $\mathrm{Kg}$ & $C_{2 C f b-a}$ \\
\hline Produtividade (em kg) de fb de Tanzânia por ha p/ vaca Cz (nas águas) & 58 & $\mathrm{Kg}$ & $C_{3 C f b-a}$ \\
\hline Produtividade (em kg) de fb de braquiária por ha p/ vaca Cz (nas águas) & 59 & $\mathrm{Kg}$ & $C_{4 C f b-a}$ \\
\hline Consumo (em kg) semestral de matéria seca (ms) por vaca Cz (na seca) & 60 & $\mathrm{Kg}$ & $C_{1 C m s-b}$ \\
\hline Produtividade (em kg) de ms de cana por ha p/ vaca Cz (na seca) & 61 & $\mathrm{Kg}$ & $C_{2 C m s-b}$ \\
\hline Produtividade (em kg) de ms de Tanzânia por ha p/ vaca Cz (na seca) & 62 & $\mathrm{Kg}$ & $C_{3 C m s-b}$ \\
\hline Produtividade (em kg) de ms de braquiária por ha p/ vaca Cz (na seca) & 63 & $\mathrm{Kg}$ & $C_{4 C m s-b}$ \\
\hline Quantidade (em kg) de ms/kg de silagem própria p/ vaca Cz (na seca) & 64 & $\mathrm{Kg}$ & $C_{5 \text { Cms-b }}$ \\
\hline Quantidade (em kg) de ms/kg de silagem comprada p/ vaca Cz (na seca) & 65 & $\mathrm{Kg}$ & $C_{6 C m s-b}$ \\
\hline Consumo (em kcal) semestral de energia (en) por vaca Cz (na seca) & 66 & Kcal & \\
\hline Produtividade (em kcal) de en de cana por ha p/ vaca Cz (na seca) & 67 & Kcal & \\
\hline Produtividade (em kcal) de en de Tanzânia por ha p/ vaca Cz (na seca) & 68 & Kcal & $c_{3 C e n-b}$ \\
\hline Produtividade (em kcal) de en de braquiária por ha p/ vaca Cz (na seca) & 69 & Kcal & $C_{4 C e n-b}$ \\
\hline Quantidade (em kcal) de en/kg de silagem própria p/ vaca Cz (na seca) & 70 & Kcal & $c_{5 C e n-b}$ \\
\hline Quantidade (em kcal) de en/kg de silagem comprada p/ vaca Cz (na seca) & 71 & Kcal & $C_{6 C e n-b}$ \\
\hline Consumo (em kg) semestral de fibra (fb) por vaca Cz (na seca) & 72 & $\mathrm{Kg}$ & $C_{1 C f b-b}$ \\
\hline Produtividade (em kg) de fb de cana por ha p/ vaca Cz (na seca) & 73 & Kg & $C_{2 C f b-b}$ \\
\hline Produtividade (em kg) de fb de Tanzânia por ha p/ vaca Cz (na seca) & 74 & $\mathrm{Kg}$ & $C_{3 C f b-b}$ \\
\hline Produtividade (em kg) de fb de braquiária por ha p/ vaca Cz (na seca) & 75 & $\mathrm{Kg}$ & $C_{4 C f b-b}$ \\
\hline Quantidade (em kg) de fb/kg de silagem própria p/ vaca Cz (na seca) & 76 & $\mathrm{Kg}$ & $C_{5 C f b-b}$ \\
\hline Quantidade (em kg) de fb/kg de silagem comprada p/ vaca Cz (na seca) & 77 & $\mathrm{Kg}$ & $C_{6 C f b-b}$ \\
\hline Produtividade semestral de leite por vaca $\mathrm{Hz}$ & 78 & $\mathrm{~L}$ & $c_{1 \text { Ple }}$ \\
\hline Produtividade semestral de leite por vaca $\mathrm{Cz}$ & 79 & $\mathrm{~L}$ & $c_{2 P l e}$ \\
\hline Consumo (em $\mathrm{R} \$$ ) de ração de vaca $\mathrm{Hz}$ & 80 & $\mathrm{R} \$$ & $C_{1 R V L}$ \\
\hline Consumo (em $\mathrm{R} \$$ ) de ração de vaca $\mathrm{Cz}$ & 81 & $\mathrm{R} \$$ & $C_{2 R V L}$ \\
\hline Consumo (em $\mathrm{R} \$$ ) de produtos veterinários de vaca $\mathrm{Hz}$ & 82 & $\mathrm{R} \$$ & $c_{1 P V e t}$ \\
\hline Consumo (em R\$) de produtos veterinários de vaca Cz & 83 & $\mathrm{R} \$$ & $C_{2 P V e t}$ \\
\hline Consumo (em R\$) de recursos p/ manutenção de pastagens p/ Hz & 84 & $\mathrm{R} \$$ & $c_{1 \mathrm{MPa}}$ \\
\hline Consumo (em R\$) de recursos p/ manutenção de pastagens p/ Hz & 85 & $\mathrm{R} \$$ & $C_{2 \mathrm{MPa}}$ \\
\hline Consumo (em R\$) de recursos p/ manutenção de equipamentos & 86 & $\mathrm{R} \$$ & $C_{M E q}$ \\
\hline Consumo (em R\$) de recursos p/ manutenção de automóvel & 87 & $\mathrm{R} \$$ & $c_{M C a}$ \\
\hline Consumo (em R\$) de sal mineral, serviços de inseminação etc. p/ Hz & 88 & $\mathrm{R} \$$ & $C_{1 S A I}$ \\
\hline Consumo (em R\$) de sal mineral, serviços de inseminação etc. p/ Cz & 89 & $\mathrm{R} \$$ & $C_{2 S A I}$ \\
\hline Salários (em R\$) para manutenção do trabalho familiar & 90 & $\mathrm{R} \$$ & $C_{M a n F a}$ \\
\hline Provisão (em R\$) para despesas com telefone & 91 & $\mathrm{R} \$$ & $C_{\text {Cte }}$ \\
\hline Provisão (em R\$) para despesas com energia elétrica & 92 & $\mathrm{R} \$$ & $c_{C e n}$ \\
\hline Provisão (em R $\$$ ) para outras despesas & 93 & $\mathrm{R} \$$ & $C_{O C}$ \\
\hline Valor de oito horas de trabalho de mão-de-obra contratada eventual & 94 & $\mathrm{R} \$$ & $C_{1 \text { cap-a }}$ \\
\hline Valor de oito horas de trabalho de mão-de-obra contratada p/ trabalhos rotina & 95 & $\mathrm{R} \$$ & $C_{2 c a p-a}$ \\
\hline Valor de aluguel de 1 hora de trator e grade & 96 & $\mathrm{R} \$$ & $C_{3 c a p-a}$ \\
\hline Valor de aluguel de 1 hora de trator e ensiladeira & 97 & $\mathrm{R} \$$ & $C_{4 c a p-a}$ \\
\hline Valor de $1 \mathrm{~kg}$ de silagem comprada $\mathrm{p} /$ vaca $\mathrm{Hz}$ & 98 & $\mathrm{R} \$$ & $C_{5 \text { cap-a }}$ \\
\hline Valor de $1 \mathrm{~kg}$ de silagem comprada $\mathrm{p} / \mathrm{vaca} \mathrm{Cz}$ & 99 & $\mathrm{R} \$$ & $C_{6 c a p-a}$ \\
\hline Valor da quantidade de ração consumida no $1^{\circ}$ semestre & 100 & $\mathrm{R} \$$ & $C_{7 \text { cap-a }}$ \\
\hline Valor dos produtos veterinários consumidos no $1^{\circ}$ semestre & 101 & $\mathrm{R} \$$ & $C_{8 c a p-a}$ \\
\hline Valor dos insumos para manutenção das pastagens no $1^{\circ}$ semestre & 102 & $\mathrm{R} \$$ & $C_{9 c a p-a}$ \\
\hline Valor da manutenção dos equipamentos no $1^{\circ}$ semestre & 103 & $\mathrm{R} \$$ & $C_{10 \text { cap- } a}$ \\
\hline Valor da manutenção do carro no $1^{\circ}$ semestre & 104 & $\mathrm{R} \$$ & $C_{11 \text { cap- } a}$ \\
\hline Valor de sal mineral, serviços veterinários etc. no $1^{\circ}$ semestre & 105 & $\mathrm{R} \$$ & $C_{12 c a p-a}$ \\
\hline Valor de salários para manutenção da família no $1^{\circ}$ semestre & 106 & $\mathrm{R} \$$ & \\
\hline Valor de serviços telefônicos no $1^{\circ}$ semestre & 107 & $\mathrm{R} \$$ & $C_{14 \text { cap- } a}$ \\
\hline Valor de energia elétrica no $1^{\circ}$ semestre & 108 & $\mathrm{R} \$$ & $C_{15 \text { cap-a }}$ \\
\hline
\end{tabular}

ENGEVISTA, v. 9, n. 1, p. 57-81, junho 2007 


\begin{tabular}{|c|c|c|c|}
\hline Valor de outras despesas de manutenção no $1^{\circ}$ semestre & 109 & $\mathrm{R} \$$ & $c_{16 \text { сар-a }}$ \\
\hline Valor da depreciação de ativos no $1^{\circ}$ semestre & 110 & $\mathrm{R} \$$ & $C_{17 c a p-a}$ \\
\hline Venda de litros de leite no $1^{\circ}$ semestre & 111 & $\mathrm{R} \$$ & $C_{18 c a p-a}$ \\
\hline Preço da cria (bezerro (a)) $\mathrm{Hz}$ & 112 & $\mathrm{R} \$$ & $C_{1 c a p-b}$ \\
\hline Preço da cria (bezerro (a)) Cz & 113 & $\mathrm{R} \$$ & $C_{2 c a p-b}$ \\
\hline Valor de 1 das seis parcelas de amortização de 1 vaca $\mathrm{Hz}$ & 114 & $\mathrm{R} \$$ & $C_{3 \text { cap-b }}$ \\
\hline Valor de 1 das seis parcelas de amortização de 1 vaca $\mathrm{Cz}$ & 115 & $\mathrm{R} \$$ & $C_{4 c a p-b}$ \\
\hline Valor corrigido a 6\%a.a. do crédito de custeio pecuário & 116 & $\mathrm{R} \$$ & $C_{5 c a p-b}$ \\
\hline Valor corrigido a $10 \%$ a.a. do crédito de custeio agrícola & 117 & $\mathrm{R} \$$ & $C_{6 c a p-b}$ \\
\hline Valor corrigido a 20\%a.a. da Cédula do Produtor Rural (CPR) & 118 & $\mathrm{R} \$$ & $C_{7 c a p-b}$ \\
\hline Valor da quantidade de ração consumida no $2^{\circ}$ semestre & 119 & $\mathrm{R} \$$ & $c_{8 \text { can-b }}$ \\
\hline Valor dos produtos veterinários consumidos no $2^{\circ}$ semestre & 120 & $\mathrm{R} \$$ & $C_{9 c a p-b}$ \\
\hline Valor dos insumos para manutenção das pastagens no $2^{\circ}$ semestre & 121 & $\mathrm{R} \$$ & $C_{10 c a p-b}$ \\
\hline Valor da manutenção dos equipamentos no $2^{\circ}$ semestre & 122 & $\mathrm{R} \$$ & $C_{11 c a p-b}$ \\
\hline Valor da manutenção do carro no $2^{\circ}$ semestre & 123 & $\mathrm{R} \$$ & $C_{12 c a p-b}$ \\
\hline Valor de sal mineral, serviços veterinários etc. no $2^{\circ}$ semestre & 124 & $\mathrm{R} \$$ & $c_{13 c a p-b}$ \\
\hline Valor de salários para manutenção da família no $2^{\circ}$ semestre & 125 & $\mathrm{R} \$$ & $c_{14 c a p-b}$ \\
\hline Valor de serviços telefônicos no $1^{\circ}$ semestre & 126 & $\mathrm{R} \$$ & $C_{15 c a p-b}$ \\
\hline Valor de energia elétrica no $1^{\circ}$ semestre & 127 & $\mathrm{R} \$$ & $C_{16 c a p-b}$ \\
\hline Valor de outras despesas de manutenção no $1^{\circ}$ semestre & 128 & $\mathrm{R} \$$ & $c_{17 c a p-b}$ \\
\hline Valor da depreciação de ativos no $2^{\circ}$ semestre & 129 & $\mathrm{R} \$$ & $C_{18 c a p-b}$ \\
\hline Venda de litros de leite no $2^{\circ}$ semestre & 130 & $\mathrm{R} \$$ & $C_{19 c a p-b}$ \\
\hline Valor corrigido a 6\%a.a. da dívida final do crédito de custeio pecuário & 131 & $\mathrm{R} \$$ & $C_{1 f}$ \\
\hline Valor corrigido a $10 \%$ a.a. da dívida final do crédito de custeio agrícola & 132 & $\mathrm{R} \$$ & $C_{2 f}$ \\
\hline Valor corrigido a 20\%a.a. da dívida final da Cédula do Produtor Rural (CPR) & 133 & $\mathrm{R} \$$ & $C_{3 f}$ \\
\hline
\end{tabular}




\section{ANEXO V}

Solução gerada pelo modelo.

\begin{tabular}{|c|c|c|c|c|c|c|c|c|c|c|c|c|}
\hline Descrição das variáveis & & An & & & Ano & & & Ano & & & Ano & \\
\hline Cultivo de milho área 1 silagem & 1 & MiSCa1y1 & 4,50 & 44 & MiSCa1y2 & 4,50 & 81 & MiSCa1y3 & 4,50 & 118 & MiSCa1y4 & 4,50 \\
\hline Cultivo de cana área $2 \mathrm{CZ}$ (kg/ha) & 2 & CaC_a2y1 & 0,76 & 45 & CaC_a2y2 & 0,76 & 82 & CaC_a2y3 & 0,76 & 119 & CaC_a2y4 & 0,76 \\
\hline Cultivo de milheto área 1 pastagem $\mathrm{Hz}$ & 3 & mily1Hz & 4,50 & 46 & mily2Hz & 4,50 & 83 & mily3Hz & 4,50 & 120 & mily $4 \mathrm{~Hz}$ & 4,50 \\
\hline Cultivo de milheto área 1 pastagem $\mathrm{Cz}$ & 4 & mily1Cz & & 47 & mily2Cz & & 84 & mily3Cz & & 121 & mily4Cz & \\
\hline Pasto Tanzânia rotacionado $\mathbf{H z}$ & 5 & Taroy1Hz & 5,00 & 48 & Taroy $2 \mathrm{~Hz}$ & 5,00 & 85 & Taroy3Hz & 5,00 & 122 & Taroy4Hz & 5,00 \\
\hline Pasto Tanzânia rotacionado $\mathrm{Cz}$ & 6 & Taroy1Cz & & 49 & Taroy $2 \mathrm{Cz}$ & & 86 & Taroy3Cz & & 123 & Taroy4Cz & \\
\hline Pasto Braquiarao Cz & 7 & pa2y01Br & 40,00 & 50 & pa2y02Br & 40,00 & 87 & pa2y03Br & 40,00 & 124 & pa2y04Br & 40,00 \\
\hline $\begin{array}{l}\begin{array}{l}\text { Orçamento de custos diretos de } \\
\text { insumos (R\$) }\end{array} \\
\end{array}$ & 8 & \$insy109 & $13.348,10$ & 51 & \$insy209 & $13.348,10$ & 88 & \$insy309 & $13.348,10$ & 125 & \$insy409 & $13.348,10$ \\
\hline Fazenda (recursos e despesas) & 9 & ReD_Fy01 & 1,00 & 52 & ReD_Fy02 & 1,00 & 89 & ReD_Fy03 & 1,00 & 126 & ReD_Fy04 & 1,00 \\
\hline $\begin{array}{l}\text { Mão-de-obra contratada (em horas) } \\
\text { eventual }\end{array}$ & 10 & MOC_ev01 & 4,85 & 53 & MOC_ev02 & 4,85 & 90 & MOC_ev03 & 4,85 & 127 & MOC_ev04 & 4,85 \\
\hline $\begin{array}{l}\text { Mão-de-obra contratada (em horas) } \\
\text { rotina }\end{array}$ & 11 & MOC_ro01 & 0,00 & 54 & MOC_ro02 & 0,00 & 91 & MOC_ro03 & & 128 & MOC_ro04 & 0,00 \\
\hline Aluguel de hora trator padrão & 12 & TrHMy01 & 37,10 & 55 & TrHMy02 & 37,10 & 92 & TrHMy03 & 37,10 & 129 & TrHMy04 & 37,10 \\
\hline Aluguel de hora trator com ensiladeira & 13 & TrHMEy01 & 16,20 & 56 & TrHMEy02 & 16,20 & 93 & TrHMEy03 & 16,20 & 130 & TrHMEy04 & 16,20 \\
\hline $\begin{array}{l}\text { Estoque Silagem Milho disponivel (kg) } \\
\mathrm{Hz}\end{array}$ & 14 & ESMy1Hz & $\begin{array}{r}169.200,0 \\
0 \\
\end{array}$ & 57 & ESMy2Hz & $\begin{array}{r}169.200,0 \\
0\end{array}$ & 94 & ESMy3Hz & $\begin{array}{r}169.200,0 \\
0 \\
\end{array}$ & 131 & ESMy4Hz & $\begin{array}{r}169.200,0 \\
0\end{array}$ \\
\hline $\begin{array}{l}\text { Estoque Silagem Milho disponivel (kg) } \\
\mathrm{Cz}\end{array}$ & 15 & ESMy1Cz & 0,00 & 58 & ESMy2Cz & & 95 & ESMy3Cz & & 132 & ESMy4Cz & \\
\hline Compra de silagem de milho para $\mathrm{Hz}$ & 16 & CSMHzy01 & $43.366,66$ & 59 & CSMHzy02 & $43.366,66$ & 96 & CSMHzy03 & $43.366,66$ & 133 & CSMHzy04 & $43.366,66$ \\
\hline Compra de silagem de milho para $\mathrm{Cz}$ & 17 & CSMCzy01 & $7.154,97$ & 60 & CSMCzy02 & $7.154,97$ & 97 & CSMCzy03 & $7.154,97$ & 134 & CSMCzy04 & $7.154,97$ \\
\hline Vaca individual holandesa $(\mathrm{Hz})$ & 18 & vacinH01 & 42,82 & & & & & & & & & $14.552,63$ \\
\hline Produção de cria Hz & 19 & Pr_criaH & 42,82 & & & & & & & & & \\
\hline Vaca individual cruzada (Cz) & 20 & vacinC01 & 42,96 & & & & & & & & & \\
\hline Produção de cria $\mathrm{Cz}$ & 21 & Pr_criaC & 42,96 & & & & & & & & & \\
\hline $\begin{array}{llll}\begin{array}{l}\text { Amortização } \\
\text { rebanho Hz }\end{array} & \text { de } & \text { investimento } & \text { no } \\
\end{array}$ & 22 & AmInH01 & 42,82 & & & & & & & & & \\
\hline
\end{tabular}




\begin{tabular}{|c|c|c|c|c|c|c|c|c|c|c|c|c|}
\hline $\begin{array}{l}\text { Amortização de investimento no } \\
\text { rebanho Cz }\end{array}$ & 23 & AmInC01 & 42,96 & & & & & & & & & \\
\hline Credito custeio pecuário & 24 & CrCPy01 & $10.015,41$ & 61 & CrCPy02 & & 98 & CrCPy03 & & 135 & CrCPy04 & \\
\hline Credito custeio agrícola & 25 & CrCIy01 & & 62 & CrCIy02 & & 99 & CrCIy03 & & 136 & CrCIy04 & \\
\hline Credito Cédula do produtor rural & 26 & CrCPRy01 & & 63 & CrCPRy02 & & 100 & CrCPRy03 & & 137 & CrCPRy04 & \\
\hline Custos de concentrado para Vaca lact. & 27 & CR_VLy01 & $56.393,28$ & 64 & CR_VLy02 & $56.393,28$ & 101 & CR_VLy03 & $56.393,28$ & 138 & CR_VLy04 & $56.393,28$ \\
\hline Custos de produtos veterinários & 28 & CPV_Ry01 & $16.774,72$ & 65 & CPV_Ry02 & $16.774,72$ & 102 & CPV_Ry03 & $16.774,72$ & 139 & CPV_Ry04 & $16.774,72$ \\
\hline Custos de manutenção de pastagens & 29 & CManPy01 & $1.482,33$ & 66 & CManPy02 & $1.482,33$ & 103 & CManPy03 & $1.482,33$ & 140 & CManPy04 & $1.482,33$ \\
\hline $\begin{array}{lll}\text { Custos de manutenção de } \\
\text { equipamentos }\end{array}$ & 30 & CManEy01 & 648,00 & 67 & CManEy02 & 648,00 & 104 & CManEy03 & 648,00 & 141 & CManEy04 & 648,00 \\
\hline Custos de manutenção de automóvel & 31 & CManCy01 & $2.689,20$ & 68 & CManCy02 & $2.689,20$ & 105 & CManCy03 & $2.689,20$ & 142 & CManCy04 & $2.689,20$ \\
\hline $\begin{array}{l}\text { Custos de sal mineral, agcbrh e } \\
\text { inseminação }\end{array}$ & 32 & CSAI_y01 & $2.174,85$ & 69 & CSAI_y02 & $2.174,85$ & 106 & CSAI_y03 & $2.174,85$ & 143 & CSAI_y04 & $2.174,85$ \\
\hline Custos de manutenção da família & 33 & CManFy01 & $13.000,00$ & 70 & CManFy02 & $13.000,00$ & 107 & CManFy03 & $13.000,00$ & 144 & CManFy04 & $13.000,00$ \\
\hline Provisão de despesas de telefone & 34 & CR_y0101 & 622,08 & 71 & CR_y0201 & 622,08 & 108 & CR_y0301 & 622,08 & 145 & CR_y0401 & 622,08 \\
\hline \begin{tabular}{llll|}
$\begin{array}{l}\text { Provisão de despesas de energia } \\
\text { elétrica }\end{array}$ & \\
\end{tabular} & 35 & CR_y0102 & $3.628,80$ & 72 & CR_y0202 & $3.628,80$ & 109 & CR_y0302 & $3.628,80$ & 146 & CR_y0402 & $3.628,80$ \\
\hline $\begin{array}{l}\text { Provisão de outras despesas de } \\
\text { manutenção }\end{array}$ & 36 & CR_y0103 & $3.389,04$ & 73 & CR_y0203 & $3.389,04$ & 110 & CR_y0303 & $3.389,04$ & 147 & CR_y0403 & $3.389,04$ \\
\hline $\begin{array}{l}\text { Depreciação ou reserva de capital } \mathbf{p} / \\
\text { repor ativos }\end{array}$ & 37 & CR_y0104 & $6.257,02$ & 74 & CR_y0204 & $6.257,02$ & 111 & CR_y0304 & $6.257,02$ & 148 & CR_y0404 & $6.257,02$ \\
\hline Venda de leite $1^{\circ}$ período & 38 & 1venleia & $\begin{array}{r}165.862,5 \\
8 \\
\end{array}$ & 75 & 2venleia & $\begin{array}{r}165.862,5 \\
8 \\
\end{array}$ & 112 & 3venleia & $\begin{array}{r}165.862,5 \\
8 \\
\end{array}$ & 149 & 4venleia & $\begin{array}{r}165.862,5 \\
8 \\
\end{array}$ \\
\hline Venda de leite $2^{\circ}$ período & 39 & 1venleib & $\begin{array}{r}165.862,5 \\
8 \\
\end{array}$ & 76 & 2venleib & $\begin{array}{r}165.862,5 \\
8 \\
\end{array}$ & 113 & 3venleib & $\begin{array}{r}165.862,5 \\
8 \\
\end{array}$ & 150 & 4venleib & $\begin{array}{r}165.862,5 \\
8 \\
\end{array}$ \\
\hline $\begin{array}{l}\text { Transferência silagem de milho para o } \\
\text { ano } \mathbf{j}+1\end{array}$ & 40 & 1Tr_ESM2 & & 77 & 2Tr_ESM3 & 0,00 & 114 & 3Tr_ESM4 & 0,00 & 151 & 4Tr_ESM5 & 0,00 \\
\hline $\begin{array}{l}\text { Transf. dinheiro do caixa jcap_\$a para } \\
\text { jcap_\$b }\end{array}$ & 41 & $1 \mathrm{TrR} \$ 1 \mathrm{~b}$ & $14.900,33$ & 78 & $2 \operatorname{TrR} \$ 2 \mathrm{~b}$ & $9.855,98$ & 115 & 3TrR\$3b & $12.910,47$ & 152 & $4 \operatorname{TrR} \$ 4 \mathrm{~b}$ & \\
\hline $\begin{array}{l}\text { Transf. dinheiro do caixa (saldo } \\
\text { positivo) } \mathrm{jb} \text { para o }(\mathrm{j}+1) \mathrm{a}\end{array}$ & 42 & $1 \operatorname{TrR} \$ 2 p$ & & 79 & $2 \operatorname{TrR} \$ 3 p$ & $17.954,82$ & 116 & $3 T r R \$ 4 p$ & & 153 & $4 \operatorname{TrR} \$ 5 p$ & \\
\hline $\begin{array}{l}\text { Transf. dinheiro do caixa (saldo } \\
\text { negativo) jb para o }(j+1) a\end{array}$ & 43 & $1 \mathrm{TDR} \$ 2 \mathrm{n}$ & & 80 & 2TDR\$3n & & 117 & $3 T D R \$ 4 n$ & $4.864,13$ & 154 & $4 T D R \$ 5 n$ & 359,02 \\
\hline
\end{tabular}




\begin{tabular}{|c|c|c|c|c|c|c|c|c|c|c|c|}
\hline \multicolumn{3}{|c|}{ Ano 05} & \multicolumn{3}{|c|}{ Ano 06} & \multicolumn{3}{|c|}{ Ano 07} & \multicolumn{3}{|c|}{ Ano 08} \\
\hline 155 & MiSCa1y5 & 4,50 & 192 & MiSCa1y6 & 4,50 & 229 & MiSCa1y7 & 4,50 & 266 & MiSCa1y8 & 4,50 \\
\hline 156 & CaC_a2y5 & 0,76 & 193 & CaC_a2y6 & 0,76 & 230 & CaC_a2y7 & 0,76 & 267 & CaC_a2y8 & 0,76 \\
\hline 157 & mily5Hz & 4,50 & 194 & mily6Hz & 4,50 & 231 & mily7Hz & 4,50 & 268 & mily8Hz & 4,50 \\
\hline 158 & mily5Cz & & 195 & mily6Cz & & 232 & mily7Cz & & 269 & mily8Cz & \\
\hline 159 & Taroy5Hz & 5,00 & 196 & Taroy6Hz & 5,00 & 233 & Taroy7Hz & 5,00 & 270 & Taroy $8 \mathrm{~Hz}$ & 5,00 \\
\hline 160 & Taroy5Cz & & 197 & Taroy6Cz & & 234 & Taroy7Cz & & 271 & Taroy8Cz & \\
\hline 161 & pa2y05Br & 40,00 & 198 & pa2y06Br & 40,00 & 235 & pa2y07Br & 40,00 & 272 & pa2y08Br & 40,00 \\
\hline 162 & Sinsy509 & $13.348,10$ & 199 & \$insy609 & $13.348,10$ & 236 & \$insy709 & $13.348,10$ & 273 & \$insy809 & $13.348,10$ \\
\hline 163 & ReD_Fy05 & 1,00 & 200 & ReD_Fy06 & 1,00 & 237 & ReD_Fy07 & 1,00 & 274 & ReD_Fy08 & 1,00 \\
\hline 164 & MOC_ev05 & 4,85 & 201 & MOC_ev06 & 4,85 & 238 & MOC_ev07 & 4,85 & 275 & MOC_ev08 & 4,85 \\
\hline 165 & MOC_ro05 & & 202 & MOC_ro06 & & 239 & MOC_ro07 & & 276 & MOC_ro08 & \\
\hline 166 & TrHMy05 & 37,10 & 203 & TrHMy06 & 37,10 & 240 & TrHMy07 & 37,10 & 277 & TrHMy08 & 37,10 \\
\hline 167 & TrHMEy05 & 16,20 & 204 & TrHMEy06 & 16,20 & 241 & TrHMEy07 & 16,20 & 278 & \begin{tabular}{|l} 
TrHMEy08 \\
\end{tabular} & 16,20 \\
\hline 168 & ESMy5Hz & $169.200,00$ & 205 & ESMy6Hz & $169.200,00$ & 242 & ESMy7Hz & $169.200,00$ & 279 & ESMy8Hz & $169.200,00$ \\
\hline 169 & ESMy5Cz & & 206 & ESMy6Cz & 0,00 & 243 & ESMy7Cz & 0,00 & 280 & ESMy8Cz & 0,00 \\
\hline 170 & CSMHzy05 & $43.366,66$ & 207 & CSMHzy06 & $43.366,66$ & 244 & CSMHzy07 & $43.366,66$ & 281 & CSMHzy08 & $43.366,66$ \\
\hline 171 & CSMCzy05 & $7.154,97$ & 208 & CSMCzy06 & $7.154,97$ & 245 & CSMCzy07 & $7.154,97$ & 282 & CSMCzy08 & $7.154,97$ \\
\hline & & 8.388,91 & & & $4.916,82$ & & & $3.835,82$ & & & \\
\hline 172 & CrCPy05 & & 209 & CrCPy06 & & 246 & CrCPy07 & & 283 & CrCPy08 & \\
\hline 173 & CrCIy05 & & 210 & CrCIy06 & & 247 & CrCIy07 & & 284 & CrCIy08 & \\
\hline 174 & CrCPRy05 & & 211 & CrCPRy06 & & 248 & CrCPRy07 & & 285 & CrCPRy08 & \\
\hline 175 & CR_VLy05 & $56.393,28$ & 212 & CR_VLy06 & $56.393,28$ & 249 & CR_VLy07 & $56.393,28$ & 286 & CR_VLy08 & $56.393,28$ \\
\hline 176 & CPV_Ry05 & $16.774,72$ & 213 & CPV_Ry06 & $16.774,72$ & 250 & CPV_Ry07 & $16.774,72$ & 287 & CPV_Ry08 & $16.774,72$ \\
\hline 177 & CManPy05 & $1.482,33$ & 214 & CManPy06 & $1.482,33$ & 251 & CManPy07 & $1.482,33$ & 288 & CManPy08 & $1.482,33$ \\
\hline 178 & CManEy05 & 648,00 & 215 & CManEy06 & 648,00 & 252 & CManEy07 & 648,00 & 289 & CManEy08 & 648,00 \\
\hline 179 & CManCy05 & $2.689,20$ & 216 & CManCy06 & $2.689,20$ & 253 & CManCy07 & $2.689,20$ & 290 & CManCy08 & $2.689,20$ \\
\hline 180 & CSAI_y05 & $2.174,85$ & 217 & CSAI_y06 & $2.174,85$ & 254 & CSAI_y07 & $2.174,85$ & 291 & CSAI_y08 & $2.174,85$ \\
\hline 181 & CManFy05 & $13.000,00$ & 218 & CManFy06 & $13.000,00$ & 255 & CManFy07 & $13.000,00$ & 292 & CManFy08 & $13.000,00$ \\
\hline 182 & CR_y0501 & 622,08 & 219 & CR_y0601 & 622,08 & 256 & CR_y0701 & 622,08 & 293 & CR_y0801 & 622,08 \\
\hline 183 & CR_y0502 & $3.628,80$ & 220 & CR_y0602 & $3.628,80$ & 257 & CR_y0702 & $3.628,80$ & 294 & CR_y0802 & $3.628,80$ \\
\hline 184 & CR_y0503 & $3.389,04$ & 221 & CR_y0603 & $3.389,04$ & 258 & CR_y0703 & $3.389,04$ & 295 & CR_y0803 & $3.389,04$ \\
\hline
\end{tabular}




\begin{tabular}{|c|c|c|c|c|c|c|c|c|c|c|c|}
\hline 185 & CR_y0504 & $6.257,02$ & 222 & |CR_y0604 & $6.257,02$ & 259 & CR_y0704 & $6.257,02$ & 296 & CR_y0804 & $6.257,02$ \\
\hline 186 & 5venleia & $165.862,58$ & 223 & 6venleia & $165.862,58$ & 260 & 7venleia & $165.862,58$ & 297 & 8venleia & $165.862,58$ \\
\hline 187 & 5venleib & $165.862,58$ & 224 & 6venleib & $165.862,58$ & 261 & 7venleib & $165.862,58$ & 298 & 8venleib & $165.862,58$ \\
\hline 188 & 5Tr_ESM6 & & 225 & 6Tr_ESM7 & & 262 & 7Tr_ESM8 & & 299 & 8Tr_ESM9 & \\
\hline 189 & $5 \operatorname{TrR} \$ 5 \mathrm{~b}$ & & 226 & $6 \operatorname{TrR} \$ 6 \mathrm{~b}$ & $3.493,58$ & 263 & $7 \operatorname{TrR} \$ 7 \mathrm{~b}$ & & 300 & $8 \operatorname{TrR} \$ 8 \mathrm{~b}$ & \\
\hline 190 & $5 \operatorname{TrR} \$ 6 p$ & $6.606,64$ & 227 & $6 \operatorname{TrR} \$ 7 p$ & $10.828,57$ & 264 & 7TrR\$8p & $16.820,60$ & 301 & $8 \operatorname{TrR} \$ 9 p$ & \\
\hline 191 & 5TDR $\$ 6 n$ & & 228 & 6TDR\$7n & & 265 & 7TDR\$8n & & 302 & 8TDR\$9n & $2.434,64$ \\
\hline
\end{tabular}

\begin{tabular}{|r|l|r|r|l|r|}
\hline & \multicolumn{2}{|c|}{ Ano 09 } & \multicolumn{3}{c|}{ Ano 10 } \\
\hline 303 & MiSCa1y9 & 4,50 & 340 & MiSCa1Y0 & 4,50 \\
\hline 304 & CaC_a2y9 & 0,76 & 341 & CaC_a2Y0 & 0,76 \\
\hline 305 & mily9Hz & 4,50 & 342 & mily10Hz & 4,50 \\
\hline 306 & mily9Cz & & 343 & mily10Cz & \\
\hline 307 & Taroy9Hz & 5,00 & 344 & Taroy0Hz & 5,00 \\
\hline 308 & Taroy9Cz & & 345 & Taroy0Cz & \\
\hline 309 & pa2y09Br & 40,00 & 346 & pa2Y10Br & 40,00 \\
\hline 310 & \$insy909 & $13.348,10$ & 347 & \$insY009 & $13.348,10$ \\
\hline 311 & ReD_Fy09 & 1,00 & 348 & ReD_FY10 & 1,00 \\
\hline 312 & MOC_ev09 & 4,85 & 349 & MOC_ev10 & 4,85 \\
\hline 313 & MOC_ro09 & & 350 & MOC_ro10 & \\
\hline 314 & TrHMy09 & 37,10 & 351 & TrHMY10 & 37,10 \\
\hline 315 & TrHMEy09 & 16,20 & 352 & TrHMEY10 & 16,20 \\
\hline 316 & ESMy9Hz & $169.200,00$ & 353 & ESMy10Hz & $169.200,00$ \\
\hline 317 & ESMy9Cz & & 354 & ESMy10Cz & 0,00 \\
\hline 318 & CSMHzy09 & $43.366,66$ & 355 & CSMHzY10 & $43.366,66$ \\
\hline 319 & CSMCzy09 & $7.154,97$ & 356 & CSMCzy10 & $7.154,97$ \\
\hline 320 & CrCPy09 & & 357 & CrCPY10 & \\
\hline 321 & CrCIy09 & & 358 & CrCIY10 & \\
\hline 322 & CrCPRy09 & & 359 & CrCPRY10 & \\
\hline 323 & CR_VLy09 & $56.393,28$ & 360 & CR_VLY10 & $56.393,28$ \\
\hline 324 & CPV_Ry09 & $16.774,72$ & 361 & CPV_RY10 & $16.774,72$ \\
\hline 325 & CManPy09 & $1.482,33$ & 362 & CManPY10 & $1.482,33$ \\
\hline 326 & CManEy09 & 648,00 & 363 & CManEY10 & 648,00 \\
\hline
\end{tabular}




\begin{tabular}{|r|l|r|r|l|r|}
\hline 327 & CManCy09 & $2.689,20$ & 364 & CManCY10 & $2.689,20$ \\
\hline 328 & CSAI_y09 & $2.174,85$ & 365 & CSAI_Y10 & $2.174,85$ \\
\hline 329 & CManFy09 & $13.000,00$ & 366 & CManFY10 & $13.000,00$ \\
\hline 330 & CR_y0901 & 622,08 & 367 & CR_Y1001 & 622,08 \\
\hline 331 & CR_y0902 & $3.628,80$ & 368 & CR_Y1002 & $3.628,80$ \\
\hline 332 & CR_y0903 & $3.389,04$ & 369 & CR_Y1003 & $3.389,04$ \\
\hline 333 & CR_y0904 & $6.257,02$ & 370 & CR_Y1004 & $6.257,02$ \\
\hline 334 & 9venleia & $165.862,58$ & 371 & Ovenleia & $165.862,58$ \\
\hline 335 & 9venleib & $165.862,58$ & 372 & 0venleib & $165.862,58$ \\
\hline 336 & 9Tr_ESM0 & & 373 & 0TrR\$0b & $11.665,29$ \\
\hline 337 & 9TrR\$9b & $5.790,01$ & 374 & 10TrR\$11 & $22.584,92$ \\
\hline 338 & 9TrR\$0p & $14.221,71$ & 375 & OR\$f & $22.584,92$ \\
\hline 339 & 9TDR\$0n & & & & \\
\hline
\end{tabular}

